\title{
GMPLS controlled Multi-layer Ethernet
}

Viktor Nordell

Spring, 2011

Master of Science Thesis

Stockholm, Sweden 2011

TRITA-ICT-EX-2011:8 


\section{GMPLS controlled multi-layer Ethernet}

in Partial Fulfillment of the Requirements for the Degree Master of Science in Engineering

VIKTOR NORDELL

Master's Thesis at the School of Information and Communication Technology Supervisor: Anders Gavler and Pontus Sköldström Examiner: Markus Hidell 



\begin{abstract}
This thesis presents the novel idea of using a GMPLS control plane for all flavors of an IEEE 802.1Q multi-layer Ethernet, applied to an access and aggregation network scenario. Such a network could provide better resilience, control of resource allocation, bandwidth guarantees and the possibility of having a unified control plane in an environment that includes more and more type of forwarding technologies.

It might also be possible to reuse existing equipment and provide backward compatibility for legacy systems when deploying such a GMPLS network over an existing Ethernet based network.

This was accomplished through extending GMPLS protocols by defining a new switch capability object, new label object for ML Ethernet, and the development of control plane procedures for handling scenarios specific to ML Ethernet. These extensions has been implemented and verified, along with the development of a ML Ethernet aware PCE, and the results indicates that this novel architecture is plausible and has an interesting potential.
\end{abstract}




\section{Sammanfattning}

I detta examensarbete presenteras den innovativa iden om att använda ett GMPLS kontrollplan för att styra alla varianter av IEEE 802.1Q flerlagrigt Ethernet, applicerat på ett access nät. Ett sådant nätverk kan ge bättre motståndskraft, finare kontroll av resurs allokeringar, mer bandbredds garantier samt ger möjligheten att ha ett gemensamt kontrollplan som inkluderar fler och fler dataplans tekniker.

Det är även möjligt att återanvända existerande utrustning och ge bakåtkompatibilitet åt existerande system när GMPLS lösningen appliceras på ett existerande Ethernet nätverk.

Detta var uppnått genom att utvidga GMPLS protokollen med nya definitioner för växling förmåga, nytt etikett objekt för flerlagrig Ethernet samt utveckling av procedurer för att hantera scenarion speciella för detta nya data plan. Dessa tilläggen har implementerats och verifierats, tillsammans med utveckling av en Ethernet medvetten PCE, och resultaten visar att denna innovativa lösning är möjlig och har intressant potential. 


\section{Acknowledgments}

My sincerest appreciation for the help and advice given by the staff at Acreo. A special thanks to my Acreo supervisors, Anders Gavler and Pontus Sköldström, who provided good guidance, advice and feedback. I would also like to thank Lukasz Brevka (DTU) and Roland Everljung (Acreo) for very valuable feedback and advice.

I would also like to thank my supervisor at KTH, Markus Hidell, who provided important advice and very valuable feedback on this report and planning of the thesis. Also my opponent, Thomas Karlsson, should be thanked for providing valuable input both on this report and on the presentation of this work.

And last but not least, thanks to my family and friends for your support and feedback. 


\section{Contents}

Contents iv

List of Figures

List of Tables $\quad$ ix

$\begin{array}{llr}1 & \text { Introduction } & 1\end{array}$

1.1 Objectives . . . . . . . . . . . . . . . . . . . . 1

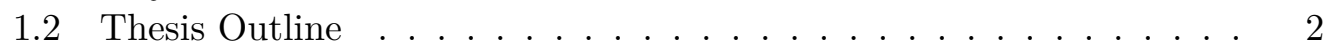

\begin{tabular}{|lll}
2 & Technology background & 3
\end{tabular}

2.1 Multi-Layer Ethernet $\ldots \ldots \ldots$. . . . . . . . . . . . . . . . . . . 4

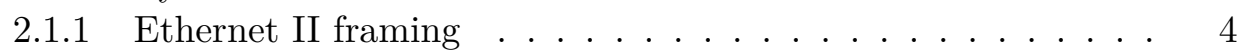

2.1 .2 IEEE $802.1 \mathrm{Q}$ VLAN Tagging . . . . . . . . . . . . . . 5

2.1.3 IEEE 802.1AD Provider Bridges (Q-in-Q) . . . . . . . . . . . 5

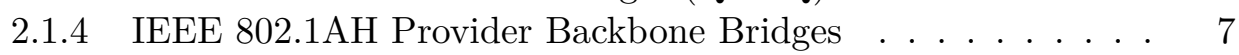

2.2 Generalized MPLS $\ldots \ldots \ldots \ldots \ldots \ldots$

2.2.1 Multiprotocol Label Switching . . . . . . . . . . . . . . . 11

$2.2 .2 \quad$ Generalized MPLS $\ldots \ldots \ldots \ldots \ldots$

$2.2 .3 \quad$ GMPLS PBB-TE $\ldots \ldots \ldots \ldots$

2.2 .4 GMPLS Path Computation . . . . . . . . . . . . . 20

2.2 .5 Shortest Path Algorithms . . . . . . . . . . . . . . . . 22

2.3 Multi-Technology Operations System Interface . . . . . . . . . . . . 23

3 Design and implementation 25

3.1 Multi-layer Ethernet Control plane . . . . . . . . . . . . . . . . . 25

$3.1 .1 \quad$ Link Switching capability . . . . . . . . . . . . . . . 25

$3.1 .2 \quad$ ML Ethernet Labell. . . . . . . . . . . . . . . . . . . . . . . . 29

3.2 Data plane implementation . . . . . . . . . . . . . . . . 30

$3.2 .1 \quad$ Data plane overview $\ldots \ldots \ldots$. . . . . . . . . . 30

3.2 .2 Data plane switch . . . . . . . . . . . . . . . 31

3.2 .3 Data plane controller . . . . . . . . . . . . . . . . . . . 33

3.3 TE-Link Announcements $\ldots \ldots \ldots \ldots$

3.3 .1 Interface specific rules $\ldots \ldots \ldots \ldots \ldots$ 
3.4 FA-LSP Setup . . . . . . . . . . . . . . . . . . 37

3.5 Port based LSPS . . . . . . . . . . . . . . . . . . . . . . . . . . . . . . . . . . . .

3.6 PCE Implementation . . . . . . . . . . . . . . . . . . . . . . 39

3.6.1 Path Computation Module . . . . . . . . . . . . . . . . . . . 40

3.6.2 Path Computation Algorithm . . . . . . . . . . . . . . . . . . 42

3.6.3 Path Validation. . . . . . . . . . . . . . . . . . . . 44

3.6.4 Virtual Topologies . . . . . . . . . . . . . . . . . . . . 46

3.7 PCE-NMS integration . . . . . . . . . . . . . . . 50

3.7 .1 MTOSI for a ML Ethernet . . . . . . . . . . . . . . . . 52

4 Verification and analysis $\quad 54$

4.1 Testbed . . . . . . . . . . . . . . . . . . . . . . 54

4.2 Demonstration network . . . . . . . . . . . . . . . . . . 55

4.3 Functional Testing . . . . . . . . . . . . . . . . . 56

4.4 PCE Performance Evaluation . . . . . . . . . . . . . . . . . . 56

4.4 .1 Time efficiency . . . . . . . . . . . . . . . . 56

4.4 .2 Memory efficiency . . . . . . . . . . . . . . . 60

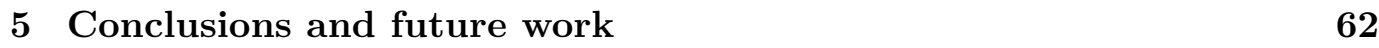

\begin{tabular}{|cc}
\hline Bibliography & 63
\end{tabular}

\begin{tabular}{|cc}
\hline Appendices & 66
\end{tabular}

\begin{tabular}{|lr}
\hline A Graph Attributes & 68 \\
\hline
\end{tabular}

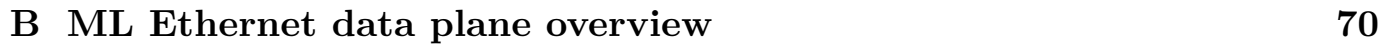




\section{List of Figures}

$2.1 \quad$ Ethernet II frame header $\ldots \ldots \ldots \ldots$. . . . . . . . . . . . . 4

2.2 A Ethernet frame header with a IEEE $802.1 \mathrm{Q}$ tag. $\ldots \ldots \ldots$. . . . . 5

2.3 The Ethernet frame with a S-tag and a C-tag. . . . . . . . . . . . . 6

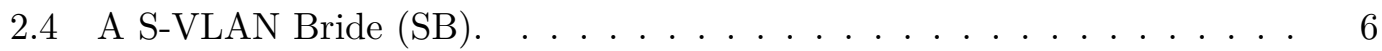

$2.5 \quad$ A Provider Edge Bridge (PEB) with customer port. The C-VLAN components switches based on the C-VID. . . . . . . . . . . 6

2.6 Overview of a simple Provider Backbone network (based on sample PBB

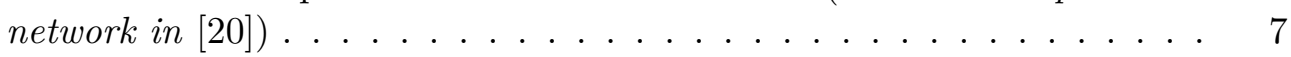

$2.7 \quad$ The Instance tagged $802.1 \mathrm{AH}$ frame header. . . . . . . . . . . . . 8

2.8 The Backbone tagged 802.1AH frame header. . . . . . . . . . . 8

2.9 The PBB I-component. $\ldots \ldots \ldots \ldots \ldots$

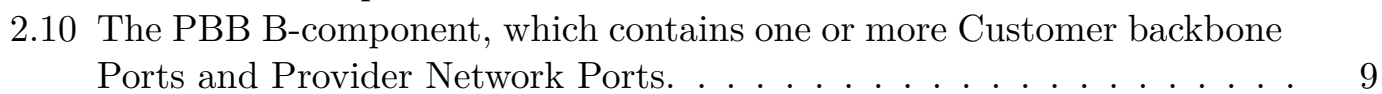

2.11 The MPLS Shim header, containing the LSP label. . . . . . . . . . . . 12

2.12 Host A sends traffic to ingress node V. This node adds the shim header and forwards the packet to LSR W. On this node a virtual link has been created. LSR W forwards the packet through this virtual link to LSR Y.

The packets finally reach the LSP egress node which removes the shim header and sends the packet to Host B. . . . . . . . . . . . . . 12

2.13 The RSVP common object header $\ldots \ldots \ldots$. . . . . . . . . . . 13

2.14 The RSVP messages between a node $\mathrm{V}$ and $\mathrm{Y}$. . . . . . . . . . . . . 14

2.15 The opaque LSA header with TE values and a sample TE TLV. . . . . 15

2.16 A GMPLS network consists of a data plane and a control plane. . . . . 16

2.17 The traffic in GMPLS flow through several type of links, this means that it is possible to tunnel one type of traffic inside another type. For example a Ethernet frame can be send inside a TDM timeslot which in turn can be sent inside a WDM wavelenght. . . . . . . . . . . . . . 17

2.18 A FA-LSP on the WDM layer exists between LSR V and Y, via LSR W and X. This FA-LSP is then used by the LSP on the L2SC layer. . . . . 17

2.19 The label used in GMPLS-PBB-TE, containing a B-VID, MAC address and currently undefined flags. 
3.1 Layer transitions in traditional GMPLS is done on the link, in multi-layer Ethernet this is done between to port on LSR. . . . . . . . . . . 26

3.2 The first version of ISCD specific information for the ML Ethernet

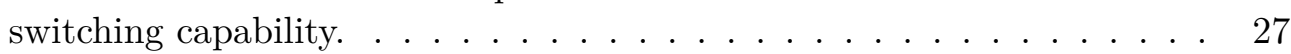

3.3 The second attempt for definition of interface switching capability spe-

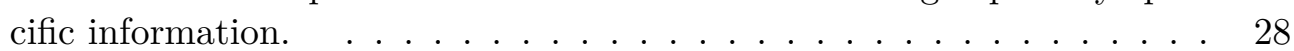

3.4 The last version of the definition. . . . . . . . . . . . . . . . . 28

3.5 The rules for a port supporting adding and removals of S-tag based on the C-tag in the received frame. . . . . . . . . . . . . . . . 29

3.6 Initial version of the ML Ethernet label. Similar to the label in GMPLSPBB-TE, but with support for I-SID values. . . . . . . . . . . . . . 29

3.7 $\quad$ The final version of ML Ethernet label, containing a field for VID/I-SID

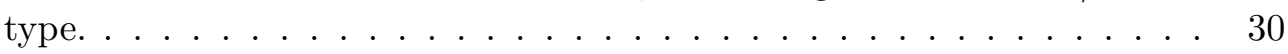

3.8 A simple multi-layer Ethernet network showing which tags are used on different links. A more detailed network can be viewed in appendix B. . 31

3.9 Sample SWSW configuration describing addition of an S-tag when entering interface "bcb1-dp" and the necessary rules for forwarding based on the S-VID value. . . . . . . . . . . . . . . . . . . . . 32

3.10 An IB-BEB switch configuration where the two FA-LSP (B-tag and ISID based) is realized on the incoming interface and not the outgoing interface. This creates a problem when announcing TE-links. . . . . . . 36

3.11 The DRAGON instance will look in the ERO and extract the FA-LSP sub-path. This sub-path will be sent to the next DRAGON instance which will redo the same procedure. $\ldots \ldots \ldots \ldots . \ldots \ldots$

3.12 The router X contains two ports capable of supporting port based LSPs. Therefore the ERO for the LSP needs to include the incoming interface. Without this information the control plane cannot know which port it

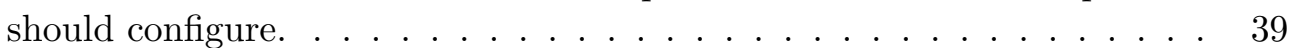

3.13 Path computation flow with some exceptions. The "Verify Computed Paths" depends on configuration and the build topology step is optimized away during performance testing. . . . . . . . . . . . . . . . 40

3.14 The network fragment contains two possible paths, path $\mathrm{X}$ and path $\mathrm{Y}$. The user has request a maximum latency of $110 \mathrm{~ms}$, this results in that only path $\mathrm{Y}$ is valid and path $\mathrm{X}$ is not. But when the optimization on line 6 of algorithm is enabled the PCE may fail to calculate the valid

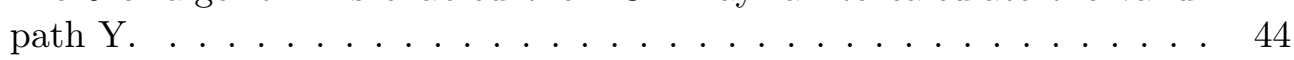

3.15 The LSP (marked in dotted red) is invalid, the tag would be added to the frame when entering and remove when exiting the router, and that layer would therefore only exist inside the router. . . . . . . . . . . . 45

3.16 Visualization (using the application Gephi[1]) of a generated network, using the Waxman model, consisting of 100 nodes. . . . . . . . . . . 46

4.1 The Ethheir testbed configuration . . . . . . . . . . . . . . 54 
4.2 Network setup used to demonstrate end-to-end allocation of QoS service in a access network. . . . . . . . . . . . . . . . . . . . 55

4.3 Mean service time, e.g. time until the client receives a response, grouped by path length. . . . . . . . . . . . . . . . 58

4.4 Graphs showing total value for each graph size. . . . . . . . . . . . . . . . 59

4.5 Graph showing two thousand request over a network with four hundred routers, both with the optimization and without. . . . . . . . . . . . 60

4.6 Visualization of RCEs heap memory usage while computing 500 requests. 61

B.1........................... . 70 


\section{List of Tables}

3.1 The defined values for the port type field in the initial version. . . . . . 27

3.2 The update frequency of different values distributed by OSPF-TE. . . . 51

$3.3 \quad$ MTOSI Layers and possible mapping to GMPLS Switch capabilities. A asterisk indicates a numerical value. . . . . . . . . . . . . . 52

$3.4 \quad$ MTOSI fields and possible mapping to OSPF-TE attributes. . . . . . . 53

A.1 Various attributes of the topology graphs used when benchmarking the RCE implementation. 


\section{Nomenclature}

\begin{tabular}{ll} 
BCB & Backbone Core Bridge \\
BEB & Backbone Edge Bridge \\
CBP & Customer backbone Port \\
CFI & Canonical Format Indicator \\
CFM & Connectivity Fault Management \\
CNP & Customer Network Port \\
CTP & Connectionless Termination Port \\
DIX & DEC-Intel-Xerox \\
ESP & Ethernet Switched Paths \\
FA-LSP & Forwarding Adjacency LSP \\
FTP & Floating Termination Point \\
GMPLS & Generalized Multiprotocol Label Switching \\
I-SID & Instance Service Identifier \\
IACD & Interface Adaptation Capability Description \\
IGP & Interior gateway protocol \\
ISCD & Interface Switching Capability Description \\
ISP & Internet Service Provider \\
L2SC & Layer-2 Switch Capable \\
LSA & Link State Advertisements \\
LSC & Lambda-Switch Capable \\
LSP & Hierarchical LSP \\
\hline
\end{tabular}




\begin{tabular}{|c|c|}
\hline ML & Multi-Layer \\
\hline MPLS & Multiprotocol Label Switching \\
\hline MTOSI & Multi-Technology Operations Systems Interface \\
\hline NMS & Network Management Systems \\
\hline OSPF & Open Shortest Path First \\
\hline P2MP & Point-to-multipoint \\
\hline $\mathrm{P} 2 \mathrm{P}$ & Point-to-point \\
\hline PBB & Provider Backbone Bridges \\
\hline PBB-TE & Provider Backbone Bridges Traffic Engineering \\
\hline PCE & Path Computation Element \\
\hline PCP & Priority Code Point \\
\hline PIP & Provider Instance Port \\
\hline PNP & Provider Network Port \\
\hline PSC & Packet-Switch Capable \\
\hline PTP & Physical Termination Point \\
\hline QoS & Quality Of Service \\
\hline $\mathrm{SNC}$ & Subnetwork Connection \\
\hline TDM & Time-division multiplexing \\
\hline $\mathrm{TE}$ & OSPF Traffic Engineering \\
\hline $\mathrm{TE}$ & Traffic Engineering \\
\hline $\mathrm{TL}$ & Topological Link \\
\hline TPID & Tag Protocol Identifier \\
\hline UPnP-QoS & Universal Plug and Play - Quality of Service \\
\hline VID & VLAN identifier \\
\hline VIP & Virtual Instance Port \\
\hline VLAN & Virtual Lan \\
\hline WDM & Wavelength-division multiplexing \\
\hline
\end{tabular}





\section{Chapter 1}

\section{Introduction}

As Internet access becomes an integral part of everyone's life the importance of a network with high reliability and availability increases. This requires more end-toend service guarantees, such as bandwidth, latency and reliability. Starting from the end-users home network, through the internet service providers access network to the backbone network.

In the home network work is being done to provide better Quality of Service guarantees, for example through the Quality of Service extension to UPnP, known as UPnP-QoS[4]. The backbone network already provides such functionality through deployments of Multiprotocol Label Switching (MPLS) or Generalized Multiprotocol Label Switching (GMPLS) enabled networks.

However the access and aggregation network is still mostly based on best effort Ethernet solutions. This thesis explores a solution which would provide the same guarantees in the access and aggregation network and thereby make it possible for end-to-end service guarantees from the home network, through the access and aggregation, to the internet service providers Internet border router.

This thesis presents a novel solution for a GMPLS controlled multi-layer Ethernet network, including data and control plane procedures and definitions. A path computation solution for ML Ethernet is also presented. This solution provides service guarantees, high availability, and reliability in an access and aggregation network, with decreased deployment cost by reusing existing Ethernet equipment. Backwards compatibility with existing Ethernet system could also be possible.

\subsection{Objectives}

The goal of this thesis project is to investigate the feasibility of constructing GMPLS based Multi-layer Ethernet hierarchical networks. This will be done with the access network in mind. This includes design and implementation of the data plane, control plane and path computation in the network. More specifically this includes:

- Data plane label design and control plane representation of the same label. 
- Control plane design of Ethernet interfaces.

- Design for creating tunneling/encapsulation in the data plane, together with the control plane logic.

- The control plane procedures and logic.

- Path computation algorithm for this type of data plane.

- Path validation for the computed paths.

In addition the thesis should investigate the possibility of integrating a Network Management Systems (NMS) into a GMPLS network. This includes an investigation of a path computation solution which integrates both information from a NMS and the network routing solution.

\subsection{Thesis Outline}

The thesis starts with an introduction to Multi-Layer Ethernet, including a short history of Ethernet and the recent developments in Ethernet standards. That chapter gives the base knowledge for understanding the data plane described in the report.

Following the Ethernet introduction comes a brief introduction to MPLS which provides the basis for the chapter about GMPLS. The chapter about GMPLS provides an introduction to GMPLS including protocol extension and procedures. It also presents a proposed standard related to the work in the thesis. The chapter also includes an introduction to GMPLS path computation, including algorithms and other aspects of path computation in a GMPLS network.

The last introductory chapter provides a brief introduction to the Multi-Technology Operations System Interface (MTOSI) standard which is a standard interface to NMS. Possible integration of this standard with the GMPLS network is investigated as it might provide benefits such as scaling when used in an access network.

In the main chapter the design and implementation is presented. This includes design of the control plane aspect, code implementation of control and data plane and various issues concerning different data plane scenarios. The next section presents the path computation implementation and a method of generating virtual topologies which is later used in the evaluation of the path computation implementation.

The following chapter presents the evaluation of the implementation, both functional testing of the network and time and memory efficiency of the path computation implementation.

The closing chapter discusses conclusions and aspects that need further study. 


\section{Chapter 2}

\section{Technology background}

This chapter gives an introduction to the technologies used for creating the Multilayer Ethernet solution. The evolutions of the different technologies are presented and how they operate.

The data plane used in this solution is Ethernet, including all the different flavors of it. These flavors of Ethernet are common in the access network and it is therefore desirable to be able to reused Ethernet as a data plane in a managed network. The different flavors are described in the first section together with a short history of its evolution.

The multi-layer Ethernet network need a control plane solution to manage resource allocation (e.g. path setup), distribute network information and for other network operations. When selecting a control plane solution several aspects need to be considered, maturity of the solution, interoperability with existing systems and scalability are among the most important. A common control plane technology in providers backbone network is GMPLS and it is therefore beneficial to be able to interoperate with those systems, for example for providing end-to-end service allocations.

The GMPLS control plane solution fulfills all the aforementioned considerations. It makes interoperation with existing system easier, it is a mature and open standard at its core and has acceptable scaling properties. This is why GMPLS was selected as control plane for the multi-layer Ethernet data plane. The details of GMPLS is explanted in the second section.

The potential size of an access network presents a problem of GMPLS, especially for the routing protocol. The scaling of the routing protocol is affected by the amount of data need to be disseminated through the routing protocol, it is therefore advantageous to reduce this data to a minimum. One solution investigated is put some of this information into a NMS solution, primarily the information less likely to changes over time, and thereby improve the scaling of the solution. One common NMS standard is MTOSI which is briefly introduced in the last section of this chapter. 


\subsection{Multi-Layer Ethernet}

Ethernet started out as a protocol used over a shared bus, for example several devices connected over a Ethernet hub, this quickly reached a major scalability limit as the bandwidth was shared over the entire network segment. The introduction of Ethernet bridges solved this by connecting several network segments together on a protocol level to form a common network.

The large amount of broadcast messages became a problem when large scale Ethernet networks were deployed[3]; the IEEE 802.1Q standard was developed to solve this [18. It added the possibility to segment the network into several virtual networks, each identified by a Virtual LAN identifier (VID). The VID is then used for filtering traffic during forwarding.

While this solved management problems for customers this still created problems for Internet Service Providers (ISPs), as the VLAN name space was shared between customers and providers. To solve this the IEEE 802.1AD standard (also known as Provider Bridges and Q-in-Q) was introduced[17]. It added an additional VID in the header and thereby gave the provider and the customers separated VLAN name spaces.

While this solved some management problem it was still a non ideal solution for very large networks. The reason is that all the bridges needed to learn and store all the addresses of both the provider devices and all the customer devices. This limits the size of the Ethernet network as a bridge has limited memory to store all the known addresses.

The solution adopted by the IEEE was to develop the 802.1AH standard 20], also known as Provider Backbone Bridges or MAC-in-MAC. This new standard made it possible to create true separation between the customer network and the provider backbone through encapsulating the customers traffic at the edges of the backbone.

\subsubsection{Ethernet II framing}

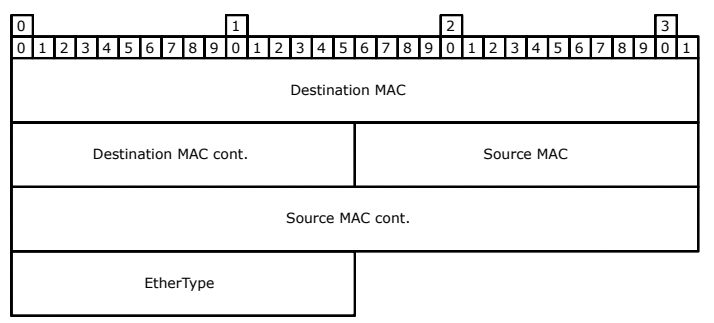

Figure 2.1: Ethernet II frame header

The original Ethernet frame consisted of destination and source MAC addresses to identify the source and destination. Followed by a 16-bit field used both to indicate size of the payload and to indicate the type of Ethernet header. This 
was necessary for keeping compatibility with IEEE 802.2. Possible because the maximum size is defined to 1518 bytes and values larger than this is interpreted as the type (called EtherType). After the EtherType/Size field comes the actual payload followed by a checksum. This original Ethernet frame is known as a DIX frame, where DIX stands for DEC-Intel-Xerox, as they together standardized this variation of Ethernet frame.

\subsubsection{IEEE 802.1Q VLAN Tagging}

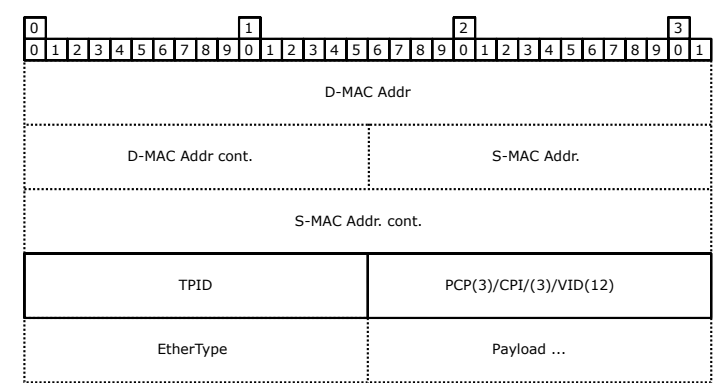

Figure 2.2: A Ethernet frame header with a IEEE 802.1Q tag.

The IEEE 802.1Q standard introduces the concept of a tag, which is inserted before the EtherType field of a frame[18]. The Tag Protocol Identifier (TPID) sits in the same place as the EtherType in the original frame and is used to identify that this is a IEEE 802.1Q tagged frame. Following is two small fields, the Priority Code Point (PCP) used to indicate the frame priority level and the Canonical Format Indicator (CFI), used to indicate what format the MAC address has. The next field is the VLAN identifier (VID) which identifies the VLAN of this frame, this VID is known as a C-VID when contained in a $802.1 \mathrm{Q}$ tag. After the tag comes the normal EtherType/Size field.

A IEEE 802.1Q enabled switch may receive either DIX frames, i.e. untagged frames, or frames with a $802.1 \mathrm{Q}$ tag. A frame can then be added to DIX frames, based on the port which the frame was received on and the tagged traffic can be forwarded out the appropriate port.

\subsubsection{IEEE 802.1AD Provider Bridges (Q-in-Q)}

A new tag, known as an S-tag, is introduced in IEEE 802.AD. This new tag contains the same fields as the $802.1 \mathrm{Q}$ tag but with a different TPID value to distinguish between the 802.1AD frame and other types of Ethernet frames. While frames may be tagged with this tag alone, it is common to tag frames first with a C-tag and then with an S-tag. 


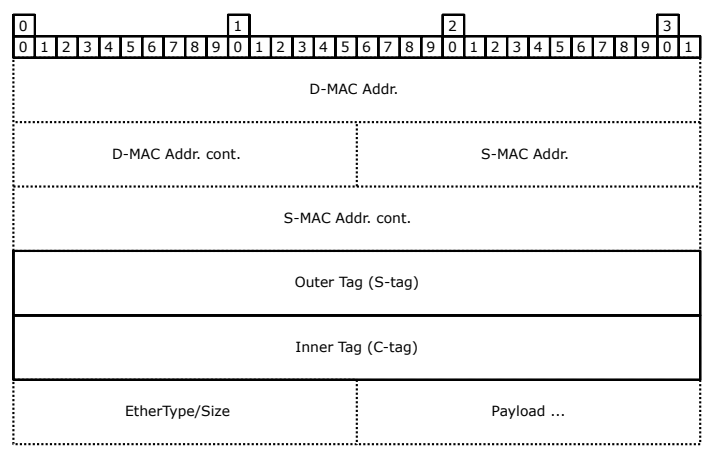

Figure 2.3: The Ethernet frame with a S-tag and a C-tag.

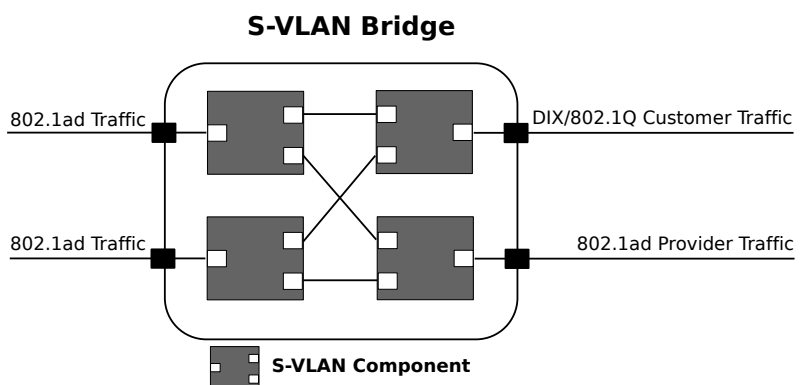

Figure 2.4: A S-VLAN Bride (SB).

Bridges capable of 802.1AD comes in two variations, S-VLAN Bridge (SB) and Provider Edge Bridge (PEB). The SB variation contains one or more S-VLAN components which switches traffic based on port or S-VID[17, it contains no component capable of understanding C-VIDs. This means that it is possible to have 802.1AD frames without the $802.1 \mathrm{Q}$ tag. The S-VLAN component may optionally translate between different S-VID values.

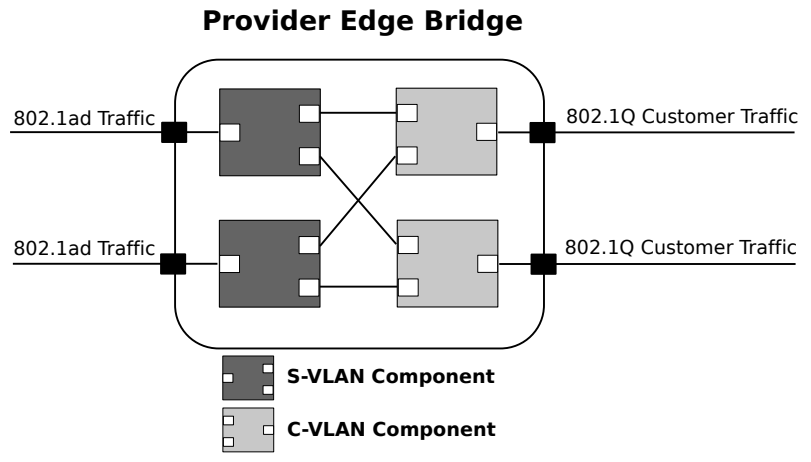

Figure 2.5: A Provider Edge Bridge (PEB) with customer port. The C-VLAN components switches based on the C-VID. 
The PEB contains one or more C-VLAN components in addition to one or more S-VLAN components, it can receive 802.1Q tagged traffic and forward the frame internally based on the C-VID to an appropriate S-VLAN component which inserts an S-tag.

\subsubsection{IEEE 802.1AH Provider Backbone Bridges}

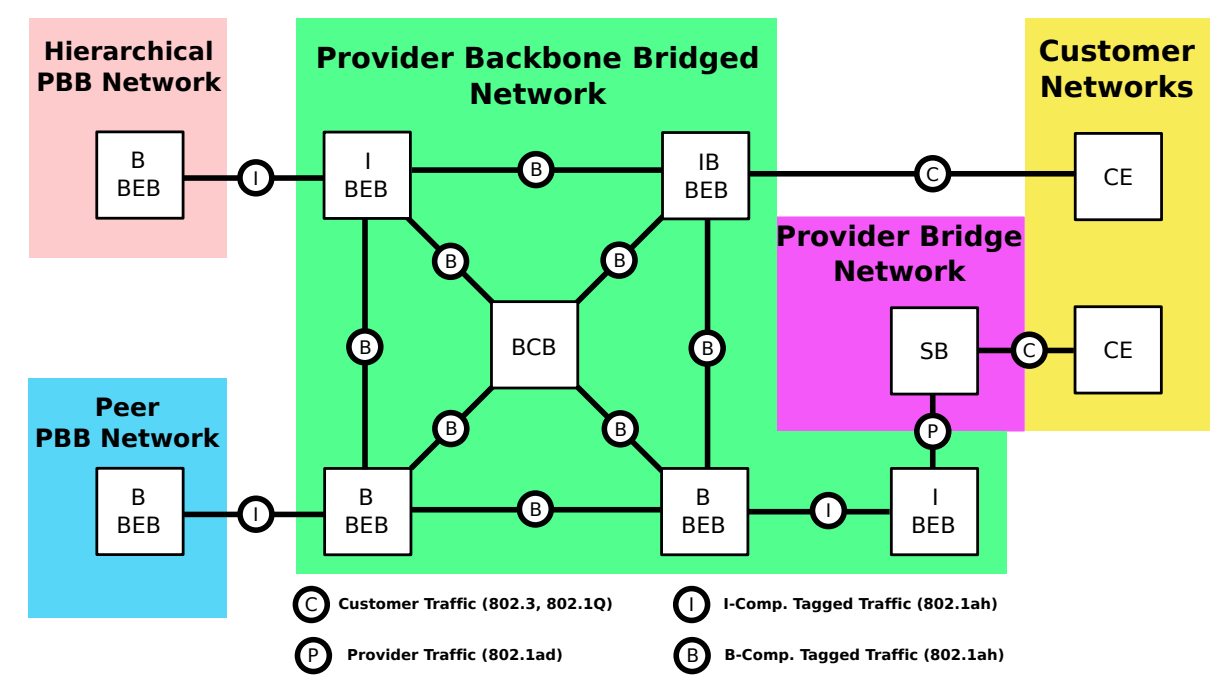

Figure 2.6: Overview of a simple Provider Backbone network (based on sample PBB network in [20])

The goal of the Provider Backbone Bridges (PBB) standard is to separate the customer network and the providers backbone, by encapsulating the traffic at the edges of the backbone network. The encapsulation means that the switches in the backbone (i.e. inside the PBB network) do not need to learn and store the MAC addresses of bridges outside of the PBB network. The traffic inside the backbone network may be encapsulated several times and thereby create a hierarchy of network layers. Both of these features means the PBB scales well and can be used in large networks.

\section{Frame Additions}

When an Ethernet frame enters a Backbone Edge Bridge (BEB) at the edge of the provider backbone network the frame is encapsulated within an I-tag. This tag contains the backbone destination and source MAC address, the TPID to identify the frame type and an Instance Service Identifier (I-SID). The I-SID is a 24-bit identifier used to differentiate between different services.

Before the frame reaches the backbone core switches the frame needs to be tagged with a Backbone tag (B-tag), this tag is identical to an S-tag in its frame 


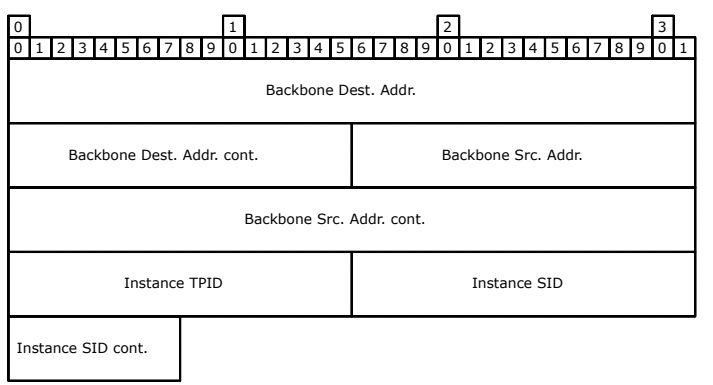

Figure 2.7: The Instance tagged 802.1AH frame header.

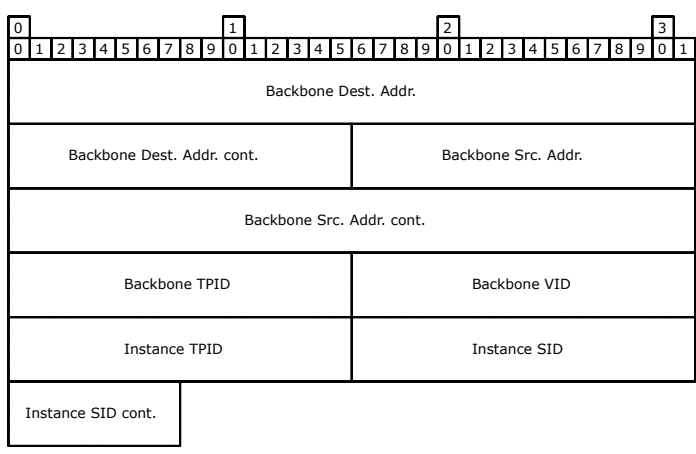

Figure 2.8: The Backbone tagged 802.1AH frame header.

definitions but differs in the usage and procedures in some cases. The addition of the B-tag may be done inside the same BEB switch or in a later step.

The core of the backbone consists of Backbone Core Bridges (BCB) which is only capable of switching on the B-tag. A normal 802.1AH switch may be configured as a BCB since the B-tag has an identical definition of the TPID and VID; this makes it possible to reuse existing hardware in the core network.

\section{Components}

The 802.AD standard contains two major types of components, the I-component and the B-component, and all the different types of PBB bridges contain at least one them. The two components contain in themselves several smaller components, each with a specific function.

\section{I-component}

The I-component categorizes incoming traffic depending on port or S-VID and assigns an I-SID to that category. It may also translate an S-VID value to an I-SID value. In the following steps it will add a backbone source and destination address (B-SA, B-DA) and lastly send the frame to a B-comp. or send it to a B-comp. based bridge. The following components resides inside the I-comp: 


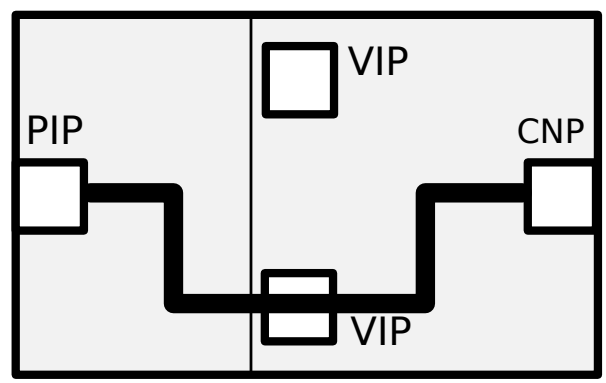

Figure 2.9: The PBB I-component.

\section{- Customer Network Port (CNP)}

The customers traffic enters this component and based on the physical port and/or S-VLAN it will forward the frame to the appropriate Virtual Instance Port (VIP).

\section{- Virtual Instance Port (VIP)}

This virtual (non-physical) port wraps the customer frame with an I-tagged header and sets the I-SID and backbone destination address (B-DA). A multicast address based on I-SID will be used if the destination address is unknown.

\section{- Provider Instance Port (PIP)}

In this last step a backbone source address (B-SA) will be set, and the frame will be forwarded either to a B-comp. or to an external switch containing a B-comp.

\section{B-component}

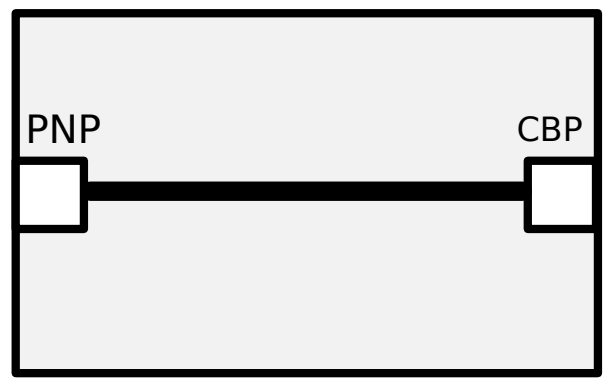

Figure 2.10: The PBB B-component, which contains one or more Customer backbone Ports and Provider Network Ports.

The B-component maps traffic to B-VLANs based on the I-SID or port number and then forwards the traffic out through the correct port. It may also translate between different destination addresses and I-SIDs. Internally the component consists of the following sub-components: 


\section{- Customer backbone Port (CBP)}

In this component the frame will be mapped to the right B-VID based on either the port or I-SID or both. It can optionally translate between different destination addresses or I-SIDs.

\section{- Provider Network Port (PNP)}

This port functions exactly as the port in IEEE 802.1AD, and switches traffic to appropriate port depending on destination address or B-VID.

\section{PBB Bridges}

The Provider Backbone Bridges standard contains several types of bridges, each with their specific area of usage. The Backbone Core Bridge is used inside the backbone network and forwards B-tagged frames. The bridge only looks at the B-tag and not the underlying client frame, which means that the bridge only needs to learn addresses of other BCBs and BEBs.

A BEB containing one or more I-components is known as an I-BEB, it receives 802.AH or $802.1 \mathrm{Q}$ framed customer traffic. The customer frame is wrapped in an I-tag header together with the appropriate I-SID and MAC addresses. This bridge will also encapsulate B-tagged traffic when received on the CNP port, which means that multiple layers of PBB can be created. The I-BEB is normally connected to B-BEBs or IB-BEBs.

If the edge bridge contains a B-component instead of I-component the bridge is known as a B-BEB. The B-comp. inside the bridge will receive I-tagged or B-tagged traffic, assign B-VLANs and optionally translate between different destination addresses and I-SID values. The traffic will then be forwarded on the appropriate port depending on B-VID and MAC address.

The IB-Backbone Edge Bridge contains both the functionality of I-BEB and $\mathrm{B}-\mathrm{BEB}$, and can therefore switch customer traffic directly on to the core backbone network.

\section{PBB Traffic Engineering}

The IEEE 802.1Qay[21] Provider Backbone Bridges Traffic Engineering (PBB-TE) standard is a recent amendment to the IEEE 802.1Q standard which includes several features required for doing traffic engineering in PBB based networks.

\section{Spanning Tree Protocol}

Ethernet bridges use the spanning tree protocol to ensure a loop free topology. This is accomplished by constructing a tree structure with each switch calculating the port used to reach the root switch and disabling all other port leading to the root. This protocol presents a problem when a control plane is used, since it will interfere with the forwarding entries set by the control plane. The PBB-TE amendment adds an option to disable the protocol for specific B-VLANs. 


\section{Ethernet Switched Paths}

The PBB-TE amendment specifies the concept of Ethernet Switched Paths (ESP), very similar to Label Switched Paths (LSP) in MPLS and GMPLS. An ESP path is identified by the tuple $<$ Backbone destination address, Backbone source address, $B-V I D>$. While both the source and destination addresses are included in the tuple only the destination address and B-VID are used to forward traffic. Each ESP is associated with an I-SID, the incoming traffic on the edges is mapped to an I-SID value which is then mapped to an ESP. The actual setup of forwarding tables in the bridges is not specified in the standard and must be done by an external agent, for example GMPLS. The upcoming standard discussed in section 2.2.3 is one example of such a solution.

\section{Protection}

The PBB-TE standard specifies the use of Connectivity Fault Management (CFM) which is standardized in IEEE 802.1ag[19]. The CFM solution can detects link and node failures that PBB-TE bridges may react on by switching to a backup path.

\subsection{Generalized MPLS}

The Generalized Multiprotocol Label Switching (GMPLS) protocol suite is a set of standardized protocol which provides a data plane agnostic control plane solution. The GMPLS suite is based on the data plane specific Multiprotocol Label Switching (MPLS) solution, and contains a set of extension to generalize the relationship between the data plane and the control plane. Two protocols provides the basis for the framework, the OSPF routing protocol with traffic engineering extensions and the RSVP resource management protocol with its respective traffic engineering extensions.

\subsubsection{Multiprotocol Label Switching}

In normal IP routing each packet is forwarded by making a longest prefix match on the destination address in the routers forwarding table. In MPLS each packet is wrapped with a label when it enters the MPLS network, and when the packet travels throughout the network the switch is doing a lookup on the label and switches the packet out from that information.

The processes of doing a longest prefix match on a IP address can today be done entirely in hardware but this was not the case during the 1990s[16. In contrast MPLS forwarding could be done entirely in hardware during the same time, which was the main motivation behind the development of MPLS. Today the main reason why MPLS is useful and popular is because of MPLS ability to do Traffic Engineering (TE) and fast failover. Traffic Engineering is the processes of control- 
ling how the traffic flows though the network, and thereby providing better Quality of Service (QOS) and better utilization of the network.

\section{Label switching}

A path in a MPLS network is called a "Label Switched Path" (LSP) and a device that forwards LSP traffic is known as a "Label Switching Router" (LSR). Each LSR maintains a look-up table used for forwarding the packet on the correct path. This table called the Label Forwarding Information Base (LFIB) and maps \{incoming interface, incoming label\} to \{outgoing interface, outgoing label\}.

\begin{tabular}{|l|l|l|l|}
\hline Label (20 bits) & Exp. (3 bits) & Stack (1 bit) & $\pi L$ (8 bits) \\
\hline
\end{tabular}

Figure 2.11: The MPLS Shim header, containing the LSP label.

All packets flowing in a MPLS network contains at least one Shim header, found between the link layer header and the IP header. This header contains a 20 bit label, 3 bit "Experimental" field, 1 bit stack flag and a TTL. The label identifies a path, the "Experimental" field is used to grade services and the stack flag is used to indicate that this shim header is the last in a stack of labels.

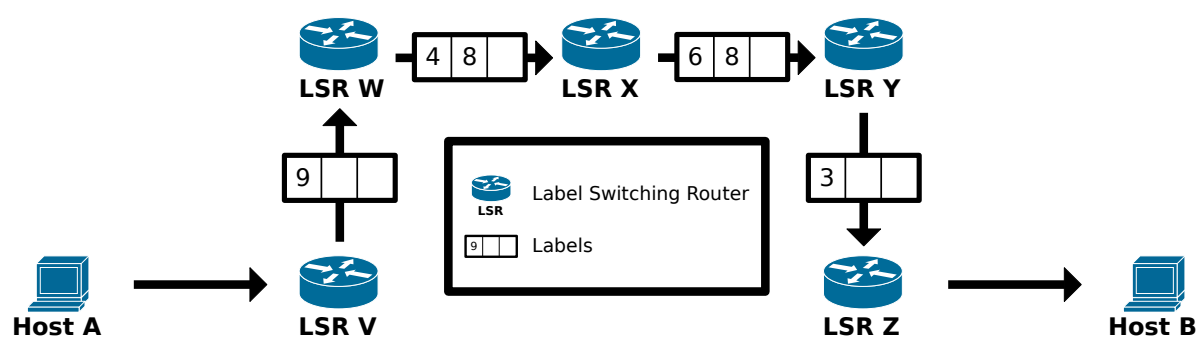

Figure 2.12: Host A sends traffic to ingress node V. This node adds the shim header and forwards the packet to LSR W. On this node a virtual link has been created. LSR W forwards the packet through this virtual link to LSR Y. The packets finally reach the LSP egress node which removes the shim header and sends the packet to Host B.

It is also possible to transmit one LSP inside another LSP. The LSP that tunnels other LSPs is then known as a hierarchical LSP (H-LSP). This is accomplished by perpending the shim header in front of the already existing shim header when entering the tunnel. When the packet is leaving the tunnel the first shim header is removed. This is an important feature which can be used to reduce signaling and improve scaling by reducing the size of the LFIB. 


\section{Protocol Extensions}

The MPLS standard extends several existing protocols to make them suitable for MPLS use. Among them are Open Shortest Path First - Traffic Engineering (OSPFTE) and Resource ReserVation Protocol - Traffic Engineering (RSVP-TE).

\section{RSVP-TE}

Before a LSP can be used the LFIB on the LSR must be populated with the appropriate path data. This could be done in several ways, by hand, via a NMS or via a label distribution protocol. One such label distribution protocol is RSVP-TE which can be used to setup and tear down LSPs[10]. The protocol is based on RSVP with several extension to accommodate for the needs of MPLS[13].

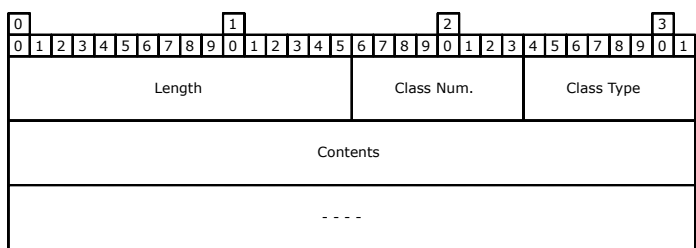

Figure 2.13: The RSVP common object header

The protocol operates by sending messages encapsulated in IP datagrams from the ingress node to the egress node, the intermediate nodes intercepts and forwards the RSVP-TE messages. A RSVP message consists of a Type-Length-Value(TLV) based packet structure which in turn contains several message objects in a classtype-length-value structure. The six top level message types involved in resource allocation is:

\section{- Path}

Sent by the ingress that wants to allocate a resource, forwarded by the nodes until it reaches the egress node which then respondes with a "Resv" message. If a node that receives a Path message cannot allocate the requested resources the node will respond with a PathErr message which will be forwarded back to the ingress node.

\section{- Resv}

The Resv message comes in response to the Path message, and message triggers the allocation of the requested resources. If the router fails to allocate the resources, for example because of bandwidth limitations, the router will send a ResvErr in response to the Resv message.

\section{- ResvConf}

Send by the ingress node to confirm the allocated path. This message serves a limited purpose in RSVP-TE. 


\section{- PathErr / ResvErr}

Error message sent as response to the Path and Resv message if the node failed to accommodate the request.

\section{- PathTear}

Sent to tear down a path.

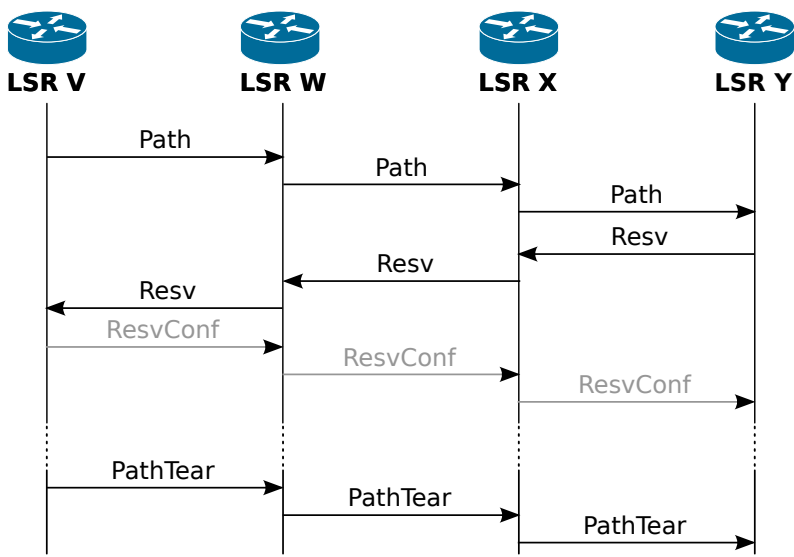

Figure 2.14: The RSVP messages between a node V and Y.

The RSVP protocol is a soft state protocol, this means that the state always needs to be refreshed, if not the path will fail. This is done by continually resending the Path and Resv message between two nodes. This creates a considerable overhead when used in a MPLS network; luckily RFC 2961 RSVP Refresh Overhead Reduction Extensions 12 reduces this problem. The Traffic Engineering extensions to RSVP contains new message objects to adapt RSVP for usage within a MPLS network.

\section{Explicit Routes}

To control the route taken in the network an Explicit Route Object (ERO) can be included in the Path message. This object includes a single header with several subobjects (encoded in a TLV format) following. Each subobject describes a hop in the path which is processed in RSVP. When RSVP receives a Path message containing an ERO the first entry must be the current node. If not, a Path Error message should be sent. RSVP then proceds by checking the next entry for the next hop. If none is present the node is the final hop in the path. This object is later extended in GMPLS to provide higher flexibility for the types of hop, as the version designed for MPLS only includes subobjects for nodes with IPv4/IPv6 based interfaces. 


\section{OSPF-TE}

Each router in a GMPLS network needs to have knowledge about the other nodes in the network; one way to do distribute this information is via a routing protocol. The protocol primarily used in MPLS networks is Open Shortest Path First (OSPF) together with the OSPF Traffic Engineering (OSPF-TE) extension [30] 23]. OSPF is an Interior gateway protocol (IGP) and a link-state routing protocol. By using OSPF each router can find a path to all the other routers in the network area.

The routing protocol OSPF functions by exchanging Link State Advertisements (LSAs) which contains information about routers, links, networks and more. Each LSA starts with a common header and a type specific payload. One such LSA is known as an opaque LSA and allows routers to share private or proprietary information across the OSPF network [14]. Routers are required to forward and store them even if the router cannot understand them.

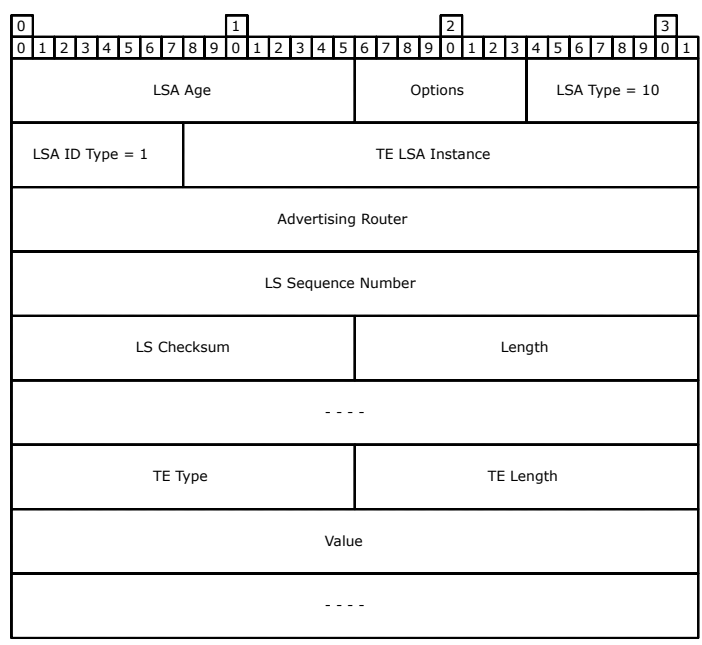

Figure 2.15: The opaque LSA header with TE values and a sample TE TLV.

The opaque TE LSA consists of a header (shown in figure 2.15) and a series of TLV objects. A TLV can in turn contain a series of TLVs. Information needed for MPLS, for example information about TE/FA-LSP links, is distributed inside those objects.

\subsubsection{Generalized MPLS}

MPLS was designed for forwarding packets and only supports this specific type of data plane. The designers of Generalized MPLS (GMPLS) wanted a control plane that could handle different type data planes, for example a Wavelength-division multiplexing (WDM) based fiber network[16]. GMPLS consists of a number of extensions to MPLS to make it more agnostic to the transport network, with a more clear separation between the control plane (which controls the data plane) and the data plane (which forwards the traffic). 


\section{Labels in GMPLS}

In MPLS all traffic was forwarded based on the shim header in front of the payload which contained the label. This label was specific to MPLS and therefore could not be used for other data planes, for example an optical data plane. Therefore the labels in GMPLS are more generalized and can be for example based on a physical property. In an optical WDM network the GMPLS label could for example be the wavelength or in Time-division multiplexing (TDM) network a timeslot.

This also means that the connection between labels and resources differs from MPLS. In MPLS the connection between label and resources is only logical. In GMPLS the relationship could be much more direct, a WDM link can only handle a fixed amount of wavelengths and there by only a limited amount of labels are available on that link. This also means that labels can have a direct relationship with the bandwidth, for example a specific wavelength can have a higher bandwidth then other wavelengths.

A GMPLS LSR can handle different types of links and therefore GMPLS standard introduced the concept switching types. All LSR interfaces in a GMPLS network have a specific switching type, for example a WDM LSR would be LambdaSwitch Capable (LSC) and a MPLS LSR would be Packet-Switch Capable (PSC).

\section{Components}

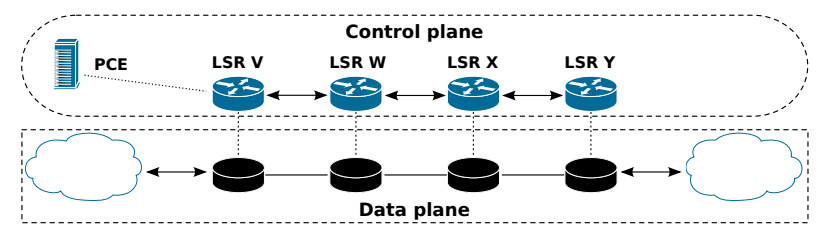

Figure 2.16: A GMPLS network consists of a data plane and a control plane.

In GMPLS there is a much more clear separation between the control plane and the data plane. A GMPLS network can have a control plane which is entirely separated from the data plane, known as pure out-of-band. It's also possible to have a GMPLS network where the control data flow in a dedicated channel of the data plane.

The LSR in a GMPLS network consists of a control part which configures the part that performs the actual data forwarding. The control part in connected to control network via a physically separated network or inside a dedicated channel of the data plane.

This Path Computation Element (PCE) device handles the computation of data plane paths. A new path could be requested on demand by a LSR or by a network management system. This element is discussed in length in section 2.2.4. 


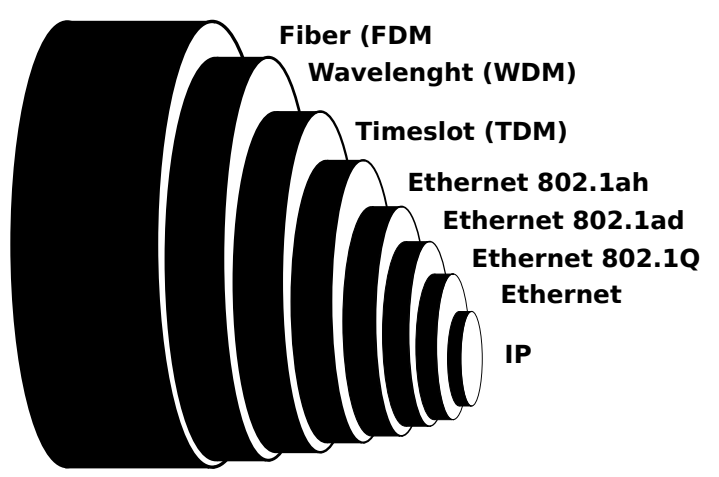

Figure 2.17: The traffic in GMPLS flow through several type of links, this means that it is possible to tunnel one type of traffic inside another type. For example a Ethernet frame can be send inside a TDM timeslot which in turn can be sent inside a WDM wavelenght.

\section{Hierarchies}

In MPLS tunnels could be created between two LSR by the use of label stacking. This is possible because there is not a direct link between the label and the physical link, this is not possible in GMPLS as a physical property can be the label. It is still possible to have tunnel via an H-LSPs, similar in concept to the MPLS H-LSP described in section 2.2.1. For example a fiber can have several wavelengths which can have several TDM timeslots. Each timeslot can then hold Ethernet traffic which contains IP traffic.

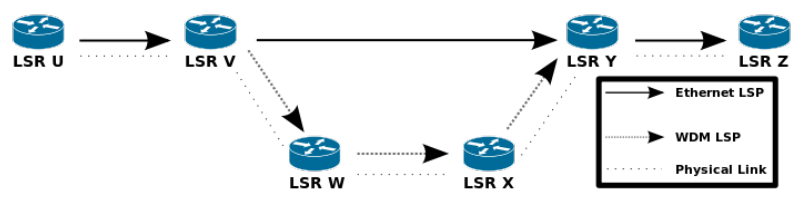

Figure 2.18: A FA-LSP on the WDM layer exists between LSR V and Y, via LSR $\mathrm{W}$ and $\mathrm{X}$. This FA-LSP is then used by the LSP on the L2SC layer.

When a new LSP is needed between two nodes in a hierarchical GMPLS network a new RSVP-TE path message is sent by the ingress LSR. This message will then be handled in the normal way until an edge node between two layers is reached, for example the edge between a Ethernet layer and an WDM layer. This node will then pause this LSPs setup and new LSP will be created over this layer, and when the new LSP is established the paused setup will be resumed. When traffic is sent between the ingress and the egress nodes the traffic will be tunneled inside the created WDM LSP. This H-LSP can also be announced as a real link by the routing protocol, this announced H-LSP will then be known as a Forwarding Adjacency LSP (FA-LSP). 


\section{Generalized RSVP-TE}

The original RSVP-TE protocol was designed for MPLS and was therefore unable to handle a more generalized data plane. Therefore several extensions were made for RSVP-TE, all designed to general in terms of the data plane used.

\section{Generalized Label Object}

Labels in MPLS may be encoded in to single 32-bit integer, while in GMPLS labels are arbitrary long and context sensitive. This means that the implementation needs to look at the switching capability value to know how to interpret the label.

\section{Enchanced Route Control}

The Explicit Route Object (ERO), used to control the route of a LSP, was defined in MPLS but was specific for IP based interfaces. Therefore this object was extended using new subobjects to support more generalized data planes. Each interface in GMPLS is assigned a 32-bit number which identifies this particular interface, this number is referred to as the interface ID. A new object containing the IP address of the router and the interface ID was introduced for use in GMPLS networks.

Also added was the ability to specify the label used for each hop. This is especially important if the label is based on physical property of the link as it requires a finer grade of control.

\section{Generalized OSPF-TE}

While OSPF-TE contained most things necessary for GMPLS a few extensions was needed when using with a more generalized data plane.

\section{Interface Switching Capability Description}

One new message object added to OSPF is the interface switching description which contains important information needed in GMPLS. Because of the generic nature of GMPLS there must be a way to describe all the interfaces a GMPLS LSR contains as each interface may be different. For example a LSR may contain a WDM interface and a Ethernet interface.

This new object, known as the Interface Switching Capability Description (ISCD), contains information about an interface in a LSR and is announced in the OSPF TE LSA. The first field is the switching capability which specifies what this interface can switch on. Each capability is assigned a number, for example Ethernet capability ("Layer-2 Switch Capable") is assigned the number 51. The assignment of numbers is designed so that it's possible to detect boundaries between layers by comparing the switching capability numbers of the two different interfaces.

Following the switching capability field come a field which specifies what type of encoding data is transmitted in, for example Lambda in case of WDM. After 16-bits 
of reserved space come eight 32-bit fields specifying the maximum bandwidth for each priority level.

The last field in the object contains information specific to the interface switching capability and is variable in size. In the case of a packet capable interface (e.g. a normal MPLS interface) this field will contain information about the minimum bandwidth for an LSP and the MTU for the interface. In this thesis this field will be called the ISCD specific field.

\section{Interface Adaptation Capability Description}

If a OSPF-TE interface advertises several ISCD entries it may be necessary to explain which ISCD should be used, and what restrictions applies, for example when determining layer borders. The Interface Adaptation Capability Description (IACD) provides this functionality, it contains a two switch capability constants, fields for bandwidth information and a field specific to the combination of switch capability constants. The two constants are organized in to an upper and lower value and this is used when determining what ISCD to use. The two switching capabilities can be seen as two internal intreconnected interfaces, which is why the bandwidth information is need. The bandwidth specifies how much bandwidth is available between these two ISCD, for example a router back plane may put a limit on the bandwidth available when using the lower or upper switching capability.

Say for example that a interface $\mathrm{X}$ on node $\mathrm{Y}$ contains two ISCDs with switch capability A and B. This interface is connected to another interface $\mathrm{Z}$ at a remote node. To figure out which of the two ISCD that should be used the outgoing interface on node $\mathrm{X}$ is looked at. The switch capability value of the outgoing interface is then matched to the upper value or the lower value of the IACD.

\section{Shared Risk Link Groups}

When several links share a resource whose failure affects all links then those links are considered to be in the same Shared Risk Link Group (SRLG). This shared resource could for example be a cable with multiple fibers and in case of cable damage it's very likely that all fibers will be affected. The use of SRLG is therefore very important in the case of protection where it's important to have a set of disjoint paths who are truly disjoint, not only in the control planes view but also in a physical sense.

To implement SRLG each interface announces a list of identifiers, where each identifier represents a shared resource that the links is a part of. The identifier is 32-bit number unique to the IGP domain.

\subsubsection{GMPLS PBB-TE}

The upcoming IETF proposed standard "GMPLS control of PBB-TE" specifies the procedures and extension to control Ethernet Switched Paths (ESPs) [15]. This enables creation of single layer LSP over a PBB backbone network. Forwarding is 
done as defined in the IEEE 802.1Qay standard, described in section 2.1.4, using the B-VID + destination MAC.

The work in this thesis is build on some parts of this upcoming standard but takes the concept further, instead of only using the B-VID the multi-layer Ethernet uses all available VLAN identifiers as well as I-SID encapsulation.

Each edge node in a GMPLS-PBB-TE network consists of IB-BEB switches where both the I-SID and the B-tag is added to the LSP payload. The I-SID is not used for forwarding but is used for identifying a service, the services are then mapped to a appropriate LSP. The mapping of I-SID to LSP can either be done manually or may use the RSVP-TE Call protocol to ensure the correct mapping between to edge nodes in the network 32 .

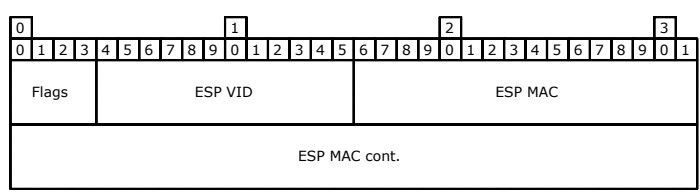

Figure 2.19: The label used in GMPLS-PBB-TE, containing a B-VID, MAC address and currently undefined flags.

The label used contains the B-VID value, a MAC address and four flags which currently are undefined. When creating P2P LSPs the address in the label represents a unicast address. The standard also defines the possibility to create P2MP LSP in which the field is a group MAC address. The label must be unique in the network domain to prevent forwarding errors.

\subsubsection{GMPLS Path Computation}

To establish an LSP in a GMPLS network a path through the network is needed. This path can be constructed by several different means. The most basic automatic method is to let the LSRs calculate the path hop by hop using the routing table. While it's easy to implement this method has a limited ability to produce a valid and optimal path. More importantly this gives operators a limited ability to do traffic engineering as the path will be based on OSPF metrics, rather than the data plane attributes and metrics.

Another solution is to have a Path Computation Element (PCE) which could be a standalone device or integrated in to an existing network element, such as an LSR. The PCE receives request for path from a source to a destination and the PCE will then calculate a path through the network based on several factors. The computation of a path maybe preformed before service provisioning, known as off-line path computation, or at the time of service provisioning, on-line path computation.

The PCE collects information about the network nodes and links and uses this information to build a Traffic Engineering Database (TED), which is used as the information backend for the path calculation. The TED may be populated from a 
number of different sources, but most commonly through the OSPF-TE information. In this thesis a NMS in explored as an secondary source for the TED.

Using the TED the PCE builds an graph which represents the GMPLS network. This graph contains the networks nodes and network links which interconnects the network nodes. This constructed graph is then used by a Shortest Path First (SPF) algorithm to calculate one or several path through the network.

\section{PCE Communication Protocol}

The Path Computation Communication Protocol (PCEP) is an IETF standardized protocol for interfacing with a PCE[37]. A client, known as a Path Computation Client (PCC), can via the protocol request paths from a PCEP enabled PCE. It may also be used between PCE instance when calculating path between domains or when Path computation is divided between several PCE instances.

The protocol operates over TCP using the IANA registered port 4189 [6] and each message is encoded in a TLV based structure with sub-objects in some messages. The protocol defines seven different message:

\section{- Open}

Sent by the PCC to the PCE to initiate a new PCEP session. The PCE will respond with a open message to acknowledge and create the session.

\section{- PCReq}

The Path Computation Request message describes a path request, including source and destination as well as constrains. This message is sent by the PCC to the PCE to initiate a path request.

\section{- PCNtf}

If the PCE or the client needs to notify the other party of an event the Path Computation Notification message is used. This message could for example be used to notify the PCC that the PCE is overloaded or to notify the PCE that the $\mathrm{PCC}$ in canceling a pending request.

\section{- PCRep}

Once the PCE has successfully calculated a path for a given request a Path Computation Response (PCRep) is sent to the client. This message includes a path between the source and destination node, but may also contain label information and other information.

\section{- PCErr}

If the PCE fails to calculate a path for a given request the PCE response with a PCE Error message. This message may also include information about the cause of the problem.

\section{- Close}

To terminate a PCEP session a close message may be sent by either party. 


\section{- Keepalive}

Sent by either party to keep a established session open.

\subsubsection{Shortest Path Algorithms}

The problem of calculating shortest paths between nodes can be categorized into four different problems.

- Single source

Find the shortest path from a source node to all other nodes.

- Single destination

From all other nodes find a path to a given node.

- Single-pair

Find the shortest path between a pair of nodes.

\section{- All-pair}

Find the shortest path between from all nodes to all nodes.

A shortest path algorithm that can solve the "All-pair" problem can also solve the other three problems as they as subsets to the "All-pair" problem. It is also important to note that a shortest path contains in itself shortest paths. This means that if we have the shortest path from A to D, via B and C, then the shortest path between $\mathrm{B}$ to $\mathrm{D}$ is also via $\mathrm{C}$.

\section{Constrained Shortest Path}

An important aspect when calculating paths in a network is that there may be constraints making certain paths or links unusable or undesirable. The reasons could be categorized in two parts: link constraints and path constraints. For example a link constraint could be the available bandwidth on the link is lower than the constraint set. A path constraint could for example be the combined latency for the entire path.

When computing paths in a GMPLS network it is necessary to consider specific attributes of the links, for example in a WDM system there might be a need to check if the wavelength of the previous WDM link is compatible with the link currently evaluated. Another aspect is maintaining the layer, if the source node has layer $\mathrm{X}$ then the layer of the destination must be the same layer X. All the layer changes in between the two nodes must also match, e.g. layer change to LSC from L2SC must be matched with the change from LSC back to L2SC.

One method for calculating paths with constraints is to verify the constrains for each potential path during path computation and thereby verify that the resulting shortest path is also valid. Another method could be to calculate alls path from the source to the destination and then validate the resulting paths, picking the shortest path. 
To speed up the path computation the topology used can be simplified by removing links that does not match the path request, known as pruning the topology. For example removing all the links that does not fulfill the bandwidth constraint.

There are also scenarios where the shortest path is not the best path. For example a path which is not the shortest path might result in bandwidth allocation which is closer to the one that was requested.

\subsection{Multi-Technology Operations System Interface}

Today's telecom operators and Internet service providers run a large number of different support systems often from different manufactures. To create a standard interface between the different systems the TM Forum created the Multi-Technology Operations Systems Interface (MTOSI) which defines a common standard for communication between these systems.

The underlying informational model of the different network elements is defined in the TMF608 standard with MTOSI implementing the actual model and the interfaces on top of it. The MTOSI standard is designed after the Service Oriented Architecture (SOA) principles with a set of web services to interact with a MTOSI system.

The web services are implemented over SOAP version 1.1 with a set of WSDL definitions for the different services. Such services are for example alarm handling, connection retrieval and equipment retrieval. Each operation returns a XML document containing the response of the request.

The specification defines three types of termination points, Physical Termination Point (PTP), Connectionless Termination Port (CTP) and Floating Termination Point (FTP). These termination points are modeled over each other, for example a CTP is modeled over a PTP. The standard also defines several other objects like connections and links.

In this thesis the MTOSI standard will be investigated as a complementary source of network information for the TED which the PCE uses for path calculations.

\section{Physical Termination Point}

The PTP maps to a physical interface, by definition without the ability to switch on the contents. Therefore a CTP or a FTP is modeled over the PTP.

\section{Connectionless Termination Port}

The CTP represents a endpoint in a Subnetwork Connection (SNC), and can be modeled on top of a PTP, CTP or another CTP.

\section{Floating Termination Point}

A FTP is termination point that cannot be directly modeled on top of a specific PTP, instead the FTP is an internal "floating" termination point. The FTP can 
for example be associated with several PTP or the underlying PTP may change depending on a set of conditions. A typical use case it to modeling protected termination points, where the underlying termination point can switch to backup in case of failure.

\section{Topological Link}

Physical Links in MTOSI are represented as a Topological Link (TL), where both endpoints are of the PTP type. This type of link may also represent a trail of other topological links or subnetwork connections.

\section{Subnetwork Connection}

In GMPLS a FA-LSP can be announced as TE-Link and seen by the PCE and other network elements as an normal network. In MTOSI the Subnetwork Connection (SNC) serves a similar function, it represents a virtual link over the network. 


\section{Chapter 3}

\section{Design and implementation}

The work consists of two parts, the design of extension and procedures, and code implementation. Since GMPLS does not support ML Ethernet several extensions and procedures were designed and later implemented in code.

The code implementation is based on six different software components, where five of the components originated in the DRAGON project but has been extended by Acreo over several years[22]. The components from the DRAGON project includes:

- NARB, a intredomin path request broker.

- RCE, a intradomin PCE.

- OSPF-TE daemon, based on OSPF from the Zebra project[27.

- RSVP-TE daemon.

- DRAGON, LSP management tool.

The last component is a software Ethernet switch constructed by Acreo. This switch, known as SWSW, implements the data plane of the implementation.

\subsection{Multi-layer Ethernet Control plane}

In the control plane two important properties need to be addressed, how represent the ML Ethernet labels and how to design ISCD for the ML Ethernet network.

\subsubsection{Link Switching capability}

The switching capability identifier in ISCD specifies what layer this interface operates on. For example layer-2 has the number 51, Lambda has 150, etc. This solution works well for clearly defined and straight forward data planes but becomes a problem for an multi-layer Ethernet for a couple of reasons.

First Ethernet has a lot of different ports that behave differently; some do simple forwarding, some add/removes tags, and some encapsulates frames. To handle this 
with the traditional switching capability scheme means that a lot of new switching capability values needs to be defined.

Most importantly there is not a strict relationship between the different the layers in Ethernet compared to traditional data planes in GMPLS. For example it is not possible to tunnel a wavelength in a TDM timeslot. But in multi-layer Ethernet it is possible to tunnel a I-tag frame with a B-tag and it is also possible to tunnel B-tag inside of a I-tag. This creates a problem when using integer constant to different layer differences, as there is no clear hierarchy between the ports.

ML Ethernet also contains a lot of different ports that behave differently; some do simple forwarding, some add/removes tags, and some encapsulates frames. To handle this with the traditional switching capability scheme means that a lot of new switching capability values needs to be defined.

\section{Layer transitions}

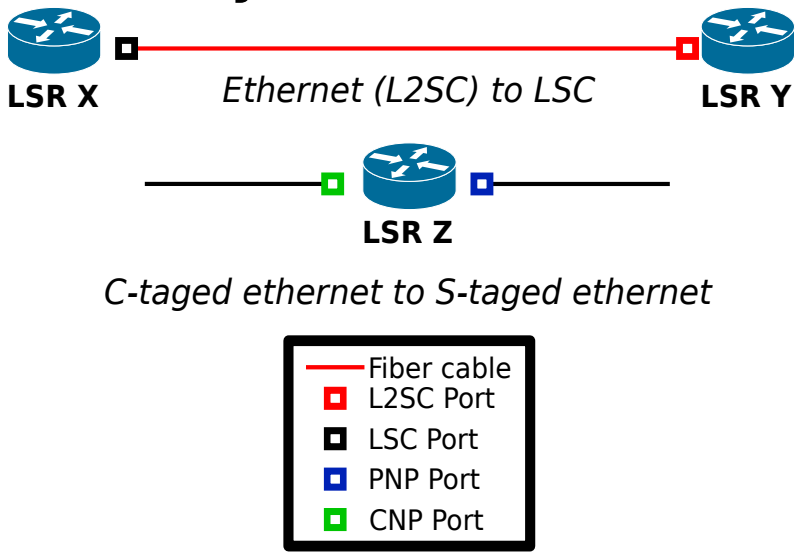

Figure 3.1: Layer transitions in traditional GMPLS is done on the link, in multilayer Ethernet this is done between to port on LSR.

The way GMPLS models layer transitions present a problem in ML Ethernet; this is modeled on links not routers. In ML Ethernet this is instead done between ports on the LSR. Consider figure 3.1, LSR X is an Ethernet switch with an optical interface. This optical interface is connected via a fiber cable to LSR Y with is an lambda capable LSR, e.g. switching traffic based on wavelengths. The transition from the Ethernet layer to lambda layer is done between the two interfaces. The LSR Z is a multi-layer Ethernet switch with a CNP port (adds S-tag based on Ctag) and a PNP port (forwards traffic based on S-tag). The transition from the C-tag layer to S-tag layer is done between the two interfaces.

In multi-layer Ethernet there may also be several layer transitions in a router, for example a switch may add both an I-tag and a B-tag. It may be possible to accomplish this in GMPLS by advertising several ISCD and one or more IACD, but this would require non standard behavior as moving from a S-tag layer to a B-tag layer requires that a I-tag is also added. This is not the case in normal GMPLS as 
moving from for example PSC to LSC does not mean that TDM is also used.

While it might be possible to solve the above mentioned problem with traditional GMPLS techniques, given some restrictions on the Ethernet network, it would be harder to implement and be less flexible. The solution chosen in this thesis is to instead define a single switching capability constant and use the switching capability specific information field to define the port and its function.

\section{Development of the definition}

The solution adopted in this work was to define a single new switching capability constant. The value 70 selected as its larger than the value for PSC (1-4) and L2SC (51) and smaller than the value for TDM (100). This is important as this is used to detect borders in a GMPLS network. The second part is how to define the switching capability specific information; this field will define the port and how it relates to the other multi-layer Ethernet ports.

\begin{tabular}{|l|l|l|}
\hline Reserved (7 bits) & Swapping (1 bit) & Port Type (16 bits) \\
\hline
\end{tabular}

Figure 3.2: The first version of ISCD specific information for the ML Ethernet switching capability.

The initial definition for the new ISCD specific information contained an eight bit long field meant for flags, with the last bit defined to indicate that this interface support label swapping. And an 16-bit long fielded identifying the port type, a sub set of the values in shown in table 3.1 .

\begin{tabular}{|l|c|l|}
\hline Name & Value & Description \\
\hline \hline 802.1Q BY PORT & 1 & IN: DIX Switch by: Port Out: $802.1 \mathrm{Q}$ \\
\hline 802.1Q BY CVID & 2 & IN: $802.1 \mathrm{Q}$ Switch by: CVID Out: $802.1 \mathrm{Q}$ \\
\hline 802.1AD BY CVID & 3 & IN: 802.1Q Switch by: CVID Out: $802 . a d+802.1 \mathrm{Q}$ \\
\hline 802.1AD BY SVID & 4 & IN: $802.1 \mathrm{Q}$ Switch by: SVID Out: $802 . a d+802.1 \mathrm{Q}$ \\
\hline 802.1AD BY PORT RAW & 5 & IN: 802.3 Switch by: Port Out: $802 . a d$ (no CVID added) \\
\hline 802.1AD BY PORT & 6 & IN: $802.1 \mathrm{Q}$ Switch by: Port Out: $802 . a d+802.1 \mathrm{Q}$ \\
\hline 802.1AH CBP BY PORT & 7 & IN: 802.3 Switch by: Port Out: $802 . a h$ B-tagged \\
\hline 802.1AH CBP BY ISID & 8 & IN: 802.3 Switch by: ISID Out: $802 . a h$ B-tagged \\
\hline 802.1AH CNP BY PORT & 9 & IN: 802.3 Switch by: Port Out: $802 . a h$ I-tagged \\
\hline 802.1AH CNP BY SVID & 10 & IN: 802.3 Switch by: Port Out: $802 . a h$ S-tagged \\
\hline
\end{tabular}

Table 3.1: The defined values for the port type field in the initial version.

This definition was inflexible, for example for each new combination of input, switching by and output a new value was needed to be added to the definition. A new definition was therefore created which offered a more flexible definition that was also less verbose. The previous 16-bit field was instead spitted in to separate 
fields. One 8-bit for port type, e.g. 802.1Q, 802.1AH, etc. And a second 8-bit field specifying what the port is switching on, e.g. none, port, CVID, etc.

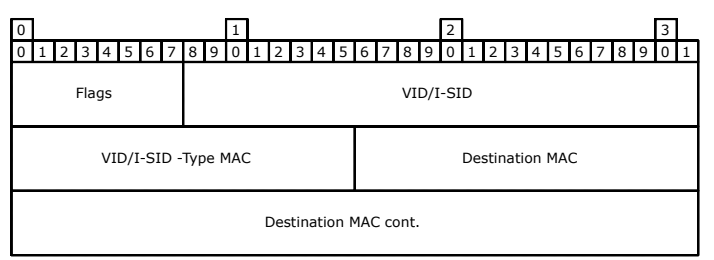

Figure 3.3: The second attempt for definition of interface switching capability specific information.

Both solutions has a serious flaw, they cannot handle switches where several steps are taken in one switch. Consider a IB-BEB switch, such a switch can be configured to add both a I-tag and an B-tag. This definition would not be able to handle such a configuration. Therefore yet another switching capability definition was developed.

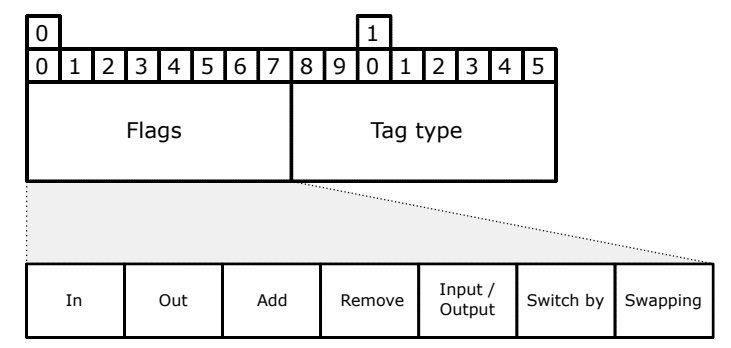

Figure 3.4: The last version of the definition.

Instead of having a fixed sized definition a variable sized list was chosen. The list contains a series of rules describing the function of the port. Each rule defines an action or capability of the port. The rule is defined as seven flags and the type of tag. This definition makes it possible to describe the input a port accepts and what it sends as output (in/out/Input/ flags). What tags is added/removed and when (in/out and add/remove flags). If the port supports label swapping ("swapping" flag) and what fields are used for forwarding decisions ("switch by" flag). Using this definition a ports behavior can be described in detail.

For example consider figure 3.5 which contains the rules needed to define a S-tag and C-tag capable PEB switch port. The first rules has the "in" flag in combination with the "switch by" and "input/output" flags set with the tag type set to C-tag. This means that the port is capable of reading and making decisions based on the $\mathrm{C}$-tag and that the port expects to receive incoming $\mathrm{C}$-tagged traffic. The second rule has the "in" and "add" flags together with the S-tag, this means that the port will add S-tag to a incoming frame. The last rule has the "out", "remove" and "input/output" flags set, which means that the port will remove the S-tag on outgoing frames. 


\begin{tabular}{|l|l|l|l|l|l|l|}
\hline In $=1$ & Out $=0$ & Add $=0$ & Remove $=0$ & Switch By $=1$ & Input/output $=1$ & Swapping $=0$ \\
\hline \multicolumn{7}{|c|}{ Tag = C-TAG } \\
\begin{tabular}{|c|c|c|l|l|l|l|}
\hline In $=1$ & Out $=0$ & Add $=1$ & Remove $=0$ & Switch By $=0$ & Input/output $=0$ & Swapping $=0$ \\
\hline In $=0$ & Out $=1$ & Add $=0$ & Remove $=1$ & Switch By $=1$ & Input/output $=1$ & Swapping $=0$ \\
\hline
\end{tabular} \\
\hline
\end{tabular}

Figure 3.5: The rules for a port supporting adding and removals of S-tag based on the $\mathrm{C}$-tag in the received frame.

This definition is also easy to extend with new Ethernet configurations, is simple to detect tag changes (important for path computation) and can be extend to support future Ethernet standards.

\subsubsection{Ethernet Label}

The label definition used in this work was inspired by the label in GMPLS PBBTE, that label contains a field for the MAC address, 12-bits for VID and four bit reserved for flags. While this might have worked for a simple S-tag based Ethernet data plane, it was not enough for a multi-layer solution. Most importantly the label lacked the bits needed for describing an I-SID value.

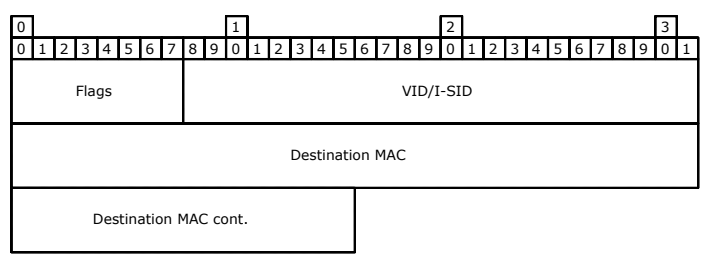

Figure 3.6: Initial version of the ML Ethernet label. Similar to the label in GMPLSPBB-TE, but with support for I-SID values.

In the first version of the label definition the field for VID/I-SID was expanded to 24-bit, to accommodate for the I-SID, compared to the PBB label. Also the flags field was expanded to accommodate more flags but also to create a better memory alignment. Three flag were defined, a "I-SID"-flag to indicate that the VID/I-SID field is a I-SID, "learning"-flag for enabling Ethernet learning for the path and a "no MAC" flag for disabling usage of the MAC address when forwarding.

During the development of the data plane controller the need for a field specifically indicating the type of VID was realized. Originally this information would be inferred from the ISCD specific information. This was done by looking through the 


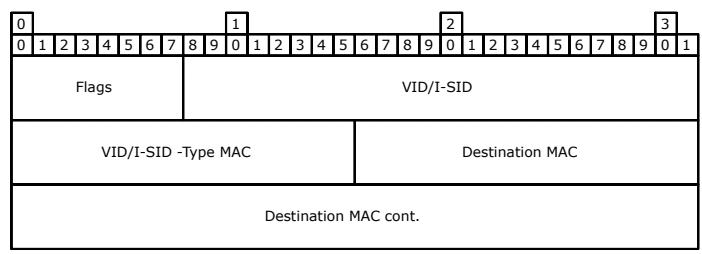

Figure 3.7: The final version of ML Ethernet label, containing a field for VID/I-SID type.

list of ISCD rules, and if the rule had one of the "switch-by"/"input"/"in"/"out" flags set, then the tag type value in the rule would be assumed to be the same as the labels VID type. Unfortunately this method was undesirable for several reasons:

1. It made it impossible to detect when an incorrect label was used, especially important during the testing/debug phase.

2. The method created major implementation problem when supporting several layer of FA-LSPs.

3. The solution was complex.

4. The method was inexact and could create situation where the assumptions were not true.

Because of these reasons the tag type field was added which solved the issues. The "is I-SID" flag was also removed as it is no longer needed.

\subsection{Data plane implementation}

The data plane implementation consists of two parts, a software based switch and a data plane controller. The data plane consists of an application running as a daemon and controlled by XML configuration. The data plane controller is implemented inside the RSVP daemon and sends configuration to the switch based on the LSP request and the interface switch capability attributes.

\subsubsection{Data plane overview}

For normal LSPs the combination of destination MAC address and VID value is used to forward frames. This is not possible when using FA-LSP, as the frames send inside the LSP may have several destination addresses. Therefore only the VID is used, and learning is used to figure out on which port a frame should be forwarded out on.

The above solution is valid for FA-LSP based on S-tags and B-tags, but when using I-SID this is not necessary as the LSP frame carried by the FA-LSP is encapsulated with the I-tag. The I-tag therefore has it's one destination address and does not need to use learning. 


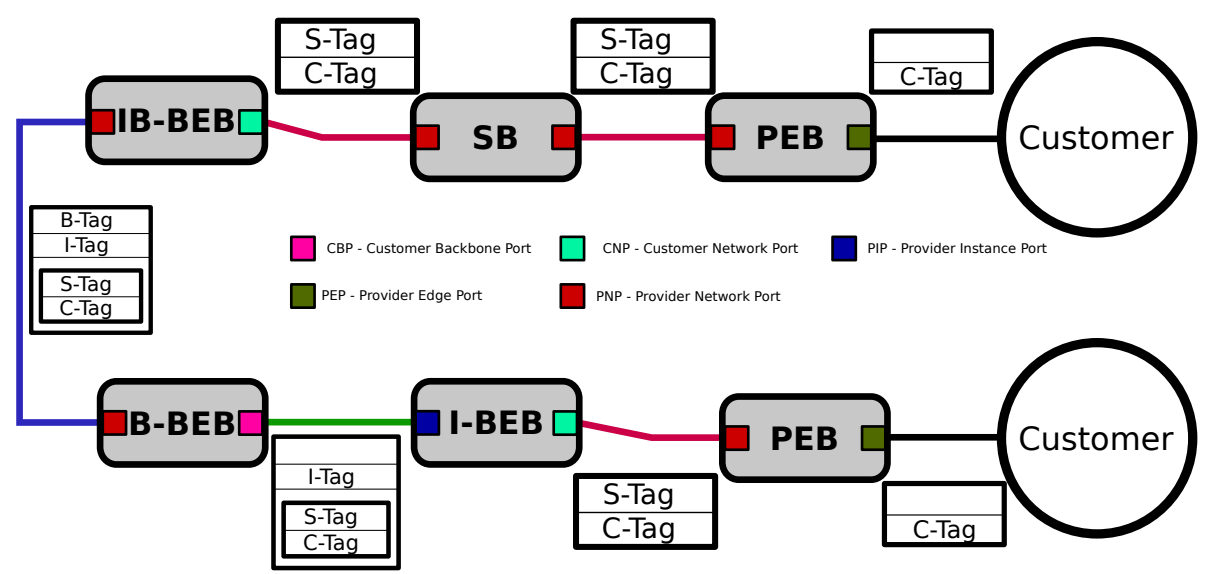

Figure 3.8: A simple multi-layer Ethernet network showing which tags are used on different links. A more detailed network can be viewed in appendix B.

Consider for example the network in Figure 3.8 this network demonstrates a simple multi-layer network using all the different tags, including I-tag encapsulation. A C-tagged frame is send from a customer node, received by a PEB based switch which adds the S-tag. This addition would therefore require a FA-LSP as this is new different Ethernet layer. Later when the frame reaches the IB-BEB switch, the frame is encapsulated in an I-tag and a B-tag is added, which also requires two new FA-LSPs. The frame will then reach the B-BEB where the B-tag is removed. This therefore terminates the B-tag based FA-LSP. When reaching the I-BEB the frame is decapsulated and the I-tag based FA-LSP is ended. Lastly the second PEB is reached and the last FA-LSP is terminated.

\subsubsection{Data plane switch}

The implementation used a highly configurable software based switch called SWSW. This software switch was developed internally at Acreo, written in $\mathrm{C}$ and runs as a daemon in Linux user space. Configuration is done by sending XML message to the running daemon or by providing a initial XML configuration file at startup.

The configuration rules consists of several section, each configuring an aspect of the switch. The sections are:

MAC-Table In this section a user defines rules for what MAC address and VIDs are associated with what port. This table is used by the switch to do lookups on which port a frame should be sent off on. When learning is enabled this table will be filled in automatically by the switch as the switch learns what MAC addresses are located at what port. If learning is disabled for a VID then all the destination MAC address needs to be present in the table for forwarding to occur. 


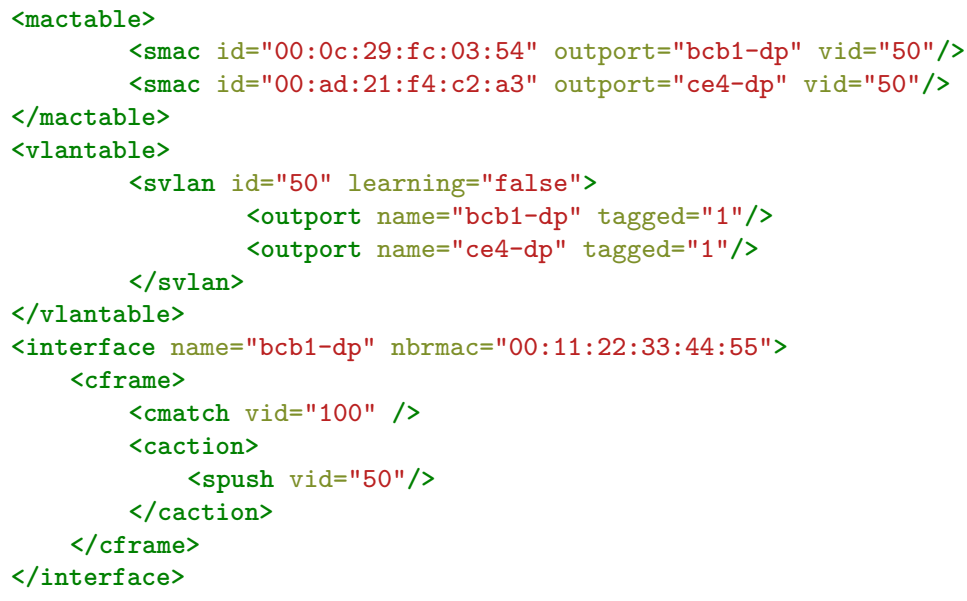

Figure 3.9: Sample SWSW configuration describing addition of an S-tag when entering interface "bcb1-dp" and the necessary rules for forwarding based on the S-VID value.

VLAN-Table This table contains information about which interfaces are associated with which VLAN. For example if the C-VLAN 11 should be enabled on interface $\mathrm{X}$ and $\mathrm{Y}$ then there need to be two rules specifying that C-VLAN 11 is enabled on interface $\mathrm{X}$ and on interface $\mathrm{Y}$. In the VLAN tag it's also specified if learning should or should not be enabled.

Interface rules In the configuration there may be several interface tags, where each tag describes some actions to be executed when a frame is received or sent out of a specified interface. The rules are nested by enclosing each tag with sub-tags. Each tag name is prefixed with the tag name, e.g. cmatch for a C-tag based match. The rules are:

- frame

Match the type of frame, for example c-tag.

- match

Used to match an attribute of the frame, mostly this is used to match the VID of a frame.

- action

A parent tag who's children contains rules to be executed. See line 14 of figure 3.9 .

- push

Add a tag $i$ front of the current one, for example add an s-tag in front of an c-tag. The VID number is also specified in an attribute of tag. 
- encap

Used to encapsulate a frame, mostly used in the I-SID case. In this tag the user specifies the source and destination MAC address and the I-SID value. These values are then used by the switch to encapsulate the frame.

The option for disable learning was not implemented in the original version and is vital to create a functional data plane. The reason is that for normal LSP both the MAC address and the VID is used for forwarding. If learning is enabled, that function will interfere with for forwarding.

Another option that was lacking in SWSW was the ability to perform action when frames are sent off from an interface. During the implementation of the data plane controller it was realized that some rule needs to be executed on the outgoing interface. This is for example the case in a IB-BEB switch where the B-tag is added when frames are sent out on the backbone interface.

\subsubsection{Data plane controller}

To configure the SWSW switch from RSVP the data plane controller was developed. This logic resides inside the RSVP daemon and is called when new RSVP message are received or sent by the daemon.

When a path message is process by the daemon it will call the allocateFreeInLabel method inside the data plane controller class, this will verify that a preferred label (if present) is available or allocate a new label. This is done by storing the VIDs currently in use.

When the reserve message is received the bindLabels method will executed. The method will construct and send configuration fragment to SWSW and in case of FA-LSP store the mapping between the physical interface and the TE-link virtual interface.

The data plane controller was the hardest part to implement and went through several iteration. A very basic controller was initially developed by Roland Elverljung at Acreo. This first implementation would only configure C-tag based forwarding rules based on the PBB label. This implementation was used as the starting point for the implementation created in this thesis.

In the second iteration the data plane controller was changed to use the ML Ethernet label, add basic support for FA-LSP and support all the different types of tags. The code to generate the XML configuration was also changed. This implementation had the following proceeding to handling LSPs:

1. Reserve message for an LSP is received by the daemon.

2. The message is parsed, verified and processed.

3. In case of FA-LSP

a) Fetch the interface ID for the TE-link from the LSP tunnel interface object. 
b) Create the ISCD for TE-link, includes creation of ISCD specific ML Ethernet rules, adjusting bandwidth. Later discussed in detail in section 3.3 .

c) Instruct OSPF to announce the TE-link.

4. The bindLabels method of the data plane controller is called:

a) If the LSP is an FA-LSP the current port, path state block, label and ISCD is stored in a FA-LSP information object. This information includes the mapping between the port and TE-port which this FA-LSP provides.

b) Checks if the port is an FA-LSP port, is so fetch the stored FA-LSP information.

c) The necessary SWSW configuration is created and sent to map the LSP in the FA-LSP (if a FA-LSP is present). This primarily includes issuing tag pushing/encapsulation configuration.

d) Insert the forwarding rules for the LSP. If this is an FA-LSP, disable learning.

e) Store the label used organized by type of tag (S-TAG, I-SID, etc.).

5. Forward the reserve message if this in not a edge node.

This solution had the major problem that it could not handle the situation when the transition to a higher layer is done an incoming interface. Consider for example an IB-BEB switch, this switch has three interfaces, one toward the backbone network and two facing a S-tagged network. Two FA-LSP are need before an S-tag based LSP can be established over the backbone network, since the IB-BEB provides both I-SID encapsulation and B-tag tagging. The problem with first iteration of the data plane controller was that the rules for mapping a LSP in to FA-LSP needed to be inserted when the first LSP using the FA-LSP was created. When the second FALSP, using the first FA-LSP, was inserted, the data plane does not know which incoming interface the final LSP will use and therefore the data plane was unable to handle this scenario.

The second attempt inserts the FA-LSP rules when a real LSP in committed and thereby can handle the problem describes above. It also simpler than the previous iteration, but depends on the fact that the data plane controller can send the same configuration to SWSW several times without problems.

1. Reserve message for an LSP is received by the daemon.

2. The message is parsed and verified.

3. In case of FA-LSP 
a) Fetch the interface ID for the TE-link from the LSP tunnel interface object.

b) Create the ISCD for TE-link, includes creation of ML Ethernet interface rules, adjusting bandwidth.

c) Instruct OSPF to announce the TE-link.

4. The bindLabels method of the data plane controller is called:

a) If the LSP is an FA-LSP stored in a FA-LSP information object including the mapping between the port and TE-port and return.

b) Fetch the ISCD for the real physical interface.

c) For each rule in the ISCD do:

i. Fetch the right label for the rule by looking in the nested FA-LSP information.

ii. If the rule is for adding a tag, find the correct label that describes the LSP that the tag will be added to.

iii. Create the XML configuration for the rule and send it to SWSW.

d) Store the label used based on the type of tag.

5. Forward the reserve message if this in not a edge node.

This implementation should be able to handle an arbitrary amount of nested FALSP.

\subsection{TE-Link Announcements}

When a new FA-LSP is created the resulting TE-link needs to be announced to the rest of the network through OSPF-TE. The process involves creating a new ISCD for the link and sending the new ISCD to the OSPF daemon who creates and advertises the new link. Most values of the new ISCD are straightforward and can be copied from the underlying interface on which the FA-LSP is created. These values include the switching capability, encoding and bandwidth. The metric can be set to the underlying interface metric minus one (to encourage use of the FA-LSP). The more complicated part is the ISCD specific part, as it must be created based on the underlying ISCD.

In first attempt the ML Ethernet rules in the ISCD was copied from the FALSPs underlying interface and then adjusted. The adjustment followed the following procedure:

1. Check if the interface has the switch capability type of ML Ethernet.

2. Loop through the rules in the ISCD specific information: 
a) Skip the first tag addition rule, as this rule is used to map the LSP to the FA-LSP.

b) Skip the first tag removal rule, for the same reason as above.

c) Adjust the tag value of the "input" and "switch by" rule to tag value minus one if the value is larger than one. This will create a TE-link interface on a level below the level of the FA-LSP.

d) Copy the row to a new list.

3. Create the TE-link via the RSVP-OSPF IPC.

The solution had the major problem that it could not handle the case when rules for entering the FA-LSP, i.e. the addition/remove of tags, exists on the incoming interface and not the outgoing interface of the switch. When this solution was run over a switch such as the on figure 3.10, the first resulting TE-link for the B-tag based FA-LSP would lack the rules for providing I-SID encapsulation.

The solution had the major issue is the case when rules for entering the FALSP, i.e. the addition/remove of tags, exists on the incoming interface and not the outgoing interface of the switch. Consider figure 3.10, two FA-LSPs is needed before any incoming LSP can be established over the interface, a B-tag based and an I-tag based. Since the switch has two possible incoming interfaces it's not known which of the interface will be used before the final LSP is established, this means that the switch configuration for creating the FA-LSP and the configuration for mapping traffic to the FA-LSP cannot be done before the first LSP is established.

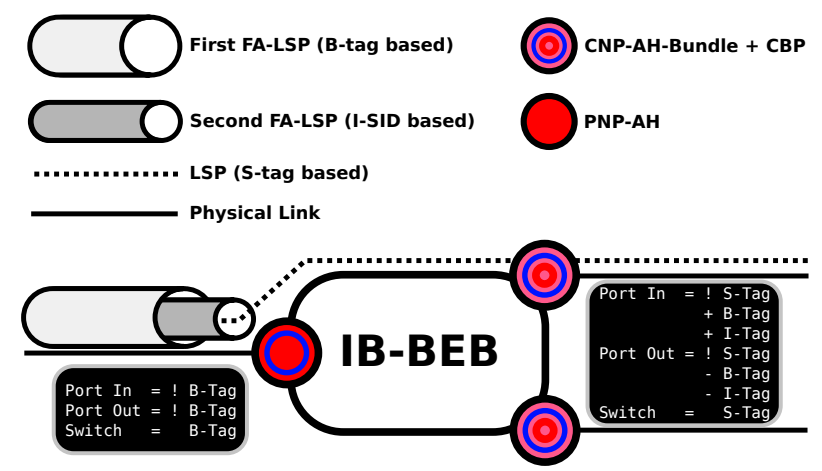

Figure 3.10: An IB-BEB switch configuration where the two FA-LSP (B-tag and I-SID based) is realized on the incoming interface and not the outgoing interface. This creates a problem when announcing TE-links.

To solve this issue the implementation firstly checks if the outgoing interface could support the FA-LSP being created. If not it will look through the rest of the interfaces on the switch the try to find which interfaces would support this FA-LSP. The ISCD of the interface found would be used as the base for the ISCD of TE-link. The procedure for adjusting the ML Ethernet interface rules is slightly different, 
the in and out flags in the rules must be flipped as the TE-link rules will add the tags when going out of the interface rather than when coming in to the interface.

A potential problem arises where the switch has several interfaces which could support the FA-LSP. But it only an issue when the possible interfaces differ in their ISCD ML Ethernet rules. The solution would be to redo the adjustment when the first LSP is created as the incoming interface is then known. However this specific logic was not implemented because of time restriction and must be considered future work.

\subsubsection{Interface specific rules}

In the previous section the scenario where the FA-LSP transition is made on the incoming interface was discussed. When the TE-link for such a FA-LSP is created it also creates a secondary issue when computing paths over that node, the rules for adding the tags now exists on two places, both the incoming physical interface and on the TE-link interface.

The proper solution for this would be create an addition ISCD for the incoming interface who's ISCD specific rules contains no tag addition rules. Unfortunately the support for multiple ISCD and IACD was untested and partly not implemented in the software framework, and therefore this was not implemented. Instead the problem was solved in the implementation by adding an addition "interface" field to the ISCD specific ML Ethernet rules. The field contains the interface number for which this rule applies. Also defined was a default value, $0 x F F F F F F F F$, to indicate that this rule applies to all outgoing interfaces that does not have a specific rule.

This new field could also be used to solve the problem where a path requires a FA-LSP inside the switch itself. This happens when the incoming interface adds a tag and the outgoing interface removes the same tag. This is not a valid path in the control plane as it's not possible to have a FA-LSP inside a single switch. However this is still possible in the data plane switch. Therefore this new field could be used to create an exception saying that it's possible to go through the interface without adding and removing the tag. This is also discussed in section 3.6 .3 of the path computation chapter.

\section{$3.4 \quad$ FA-LSP Setup}

A multi-layer Ethernet contains several layers, and when a LSP is created there may be a need to create FA-LSPs for each layer transition in the network. During the development of the implementation it became apparent that an automated solution was needed. The normal way do accomplish this is by creating new FA-LSP when the RSVP implementation detects border transitions, commonly called "triggered FA-LSPs". This solution was investigated and the unfortunate conclusion was that this would be to hard too implement within the current RSVP implementation.

Therefore another solution was developed based on automatic FA-LSP creation when committing the LSP. When a user committed a LSP in the DRAGON CLI 
the application will ask the PCE for a path, the response would include information about where the layer transitions are located. From this information DRAGON can identify which FA-LSP is needed and the end points for the FA-LSPs.

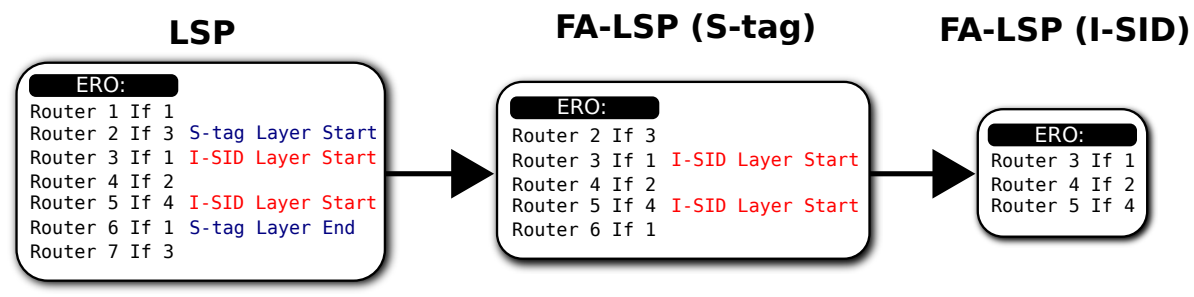

Figure 3.11: The DRAGON instance will look in the ERO and extract the FA-LSP sub-path. This sub-path will be sent to the next DRAGON instance which will redo the same procedure.

The application starts with locating the endpoints of the first layer and extracts the ERO of that sub-path. It will then proceed by connecting to the DRAGON instance of one of the endpoint of that layer. Using the value of the LSP and the sub-path the application configures the other DRAGON instance. The last step is to commit the FA-LSP on the remote instance. This remote instance will do the same steps and there by create all the FA-LSP needed for the sub-path given to it. When the remote instance is done creating the FA-LSP the local instance will replace the sub-path with the new TE-link. In the end all necessary FA-LSP will be created and the LSP can be committed.

\subsection{Port based LSPs}

Most Ethernet bridges may be configured to automatically add a predefined tag to a frame entering a port on switch, e.g. a DIX frame enters a PEB switch which automatically adds a C-tag. This could be used to create LSPs which ingress/egress port is configured to only use this LSP. Such a LSP could be very useful as it would behave as a layer-2 P2P link, for example for providing layer-2 VPN services. There may also be Ethernet bridges that only supports port based tagging.

While the current implementation does not implement specifically port based LSPs it was a something considered during the development and design of the data and control plane. The ISCD specific information described in section 3.1.1 provides a way to describe interfaces that provides port based LSPs. This could be done by setting the "switch by" flag to none, which will tell the control plane that the interface cannot switch on any Ethernet field and therefore does its switch decisions based on the port.

When using a port based LSP the traffic is tagged on the incoming port of the switch with the values from the LSP label and forwarded out on the outgoing port. This creates a problem when signaling such an LSP with RSVP-TE as the incoming port needs to be part of the ERO and needs to be considered by the implementation. 


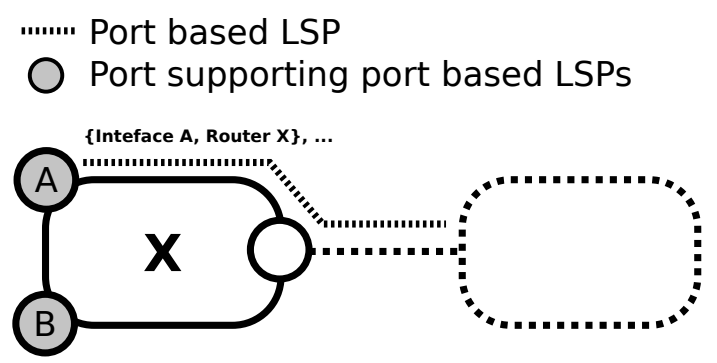

Figure 3.12: The router X contains two ports capable of supporting port based LSPs. Therefore the ERO for the LSP needs to include the incoming interface. Without this information the control plane cannot know which port it should configure.

The reason is that there may be several ports of the switch which can support a port based LSP, as seen in figure 3.12, if the incoming interface is not part of the ERO the switch cannot know which port to configure.

It is allowed (according the RFC 3209) specify the incoming interface in the ERO, but the ERO may not contain a secondary entry for the same node (e.g. \{incoming interface, node $\mathrm{X}\}$, \{outgoing interface, node $\mathrm{X}$ \}) as the aforementioned RFC specifies that the first ERO entry must be deleted in that case. After the first port based LSP is committed to the data plane the control plane will need to set the "reservable bandwidth" attribute on the link to zero to prevent additional LSPs to be created over that port. The reason for this is a port based LSP can only handle a single LSP.

Unfortunately the ERO behavior mentioned above was non trivial to implement in the RSVP implementation used, and therefore port based LSP was not implementation in thesis.

\subsection{PCE Implementation}

The Dragon software suit contains two modules, running as demons and written in $\mathrm{C}++$, who implements the PCE functionality. The NARB demon acts as a broker for intradomain and intradomin PCE requests, in this thesis NARB only forwards the requests to RCE . Both the PCEP protocol and an DRAGON specific protocol is supported for making path computation request. In this thesis only PCEP based communication was used.

The second module is known as RCE and implements the actual path computation logic. The Traffic Engineering Database is located in the RCE module, implemented as a RADIX tree with router addresses as index keys. Information from OSPF is pushed to the module as LSA messages and inserted in to the TED. The path computation logic is implemented by subclassing an abstract class and functions like a path computation logic interface. This solution makes it possible to have several separate path computation implementations which can be selected in the RCE configuration file. 
In $\mathrm{RCE}$ the process of handling a path request is as follows:

1. Receive a path request from NARB (internal IPC protocol).

2. Instantiate and call the preconfigured path computation module.

3. Verify the path request

4. Build the network graph.

5. Prune the network graph.

6. Calculate one or more paths using the path computation algorithm.

7. Select the best path based on requester preference.

8. Encode the resulting path and return response to NARB.

The majority of the thesis implementation work in the RCE has been focused on modifying an existing path computation module initial implemented by a previous thesis[29].

\subsubsection{Path Computation Module}

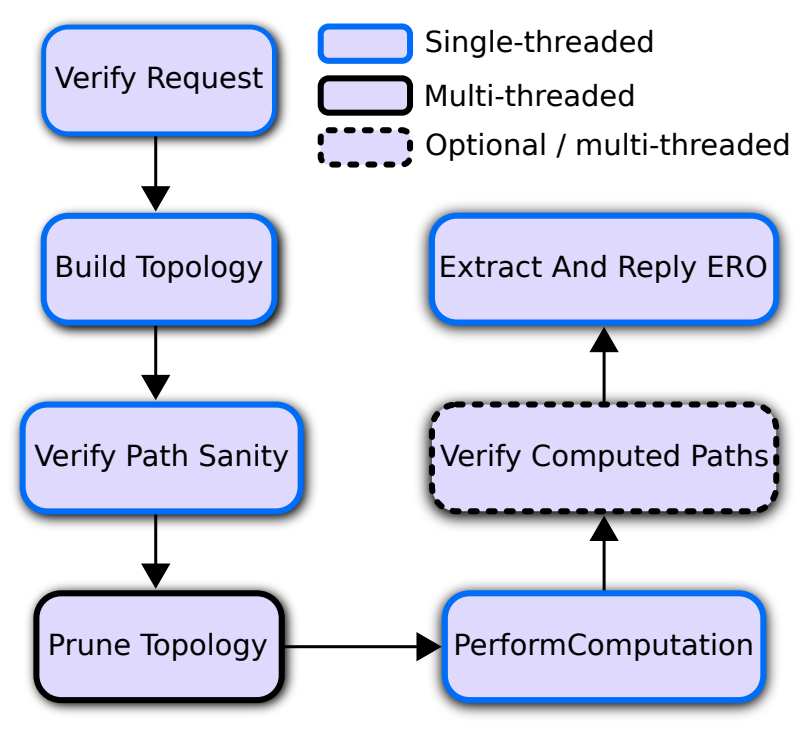

Figure 3.13: Path computation flow with some exceptions. The "Verify Computed Paths" depends on configuration and the build topology step is optimized away during performance testing.

The first step taken by path computation module is to verify the request. This is a set of basic sanity checks, including checking that the source and destination exists and that the pair is not the same node. 
Next step the in process is to build the topology from the TED, this involves constructing routers and populating the routers with the links. Each router is represented via an object which includes outgoing and incoming links, router id and other properties. Each link contains a reference to two endpoints, the switch capability / adaption capability / bandwidth and other properties for the near end of the link (relative to the router). In the stock version of RCE this step is executed on all requests, even if the TED remained unchanged since the last request, creating a considerable computational overhead. During the performance testing with a virtual network this step was optimized to only calculating this topology once and be cached, eliminating this step during performance testing.

If the previous step was successfully completed the process moves forward by doing an addition step of validating the request. In this step the logic verifies that the destination and source ends on the same layer, otherwise the request is invalid.

To simply the topology the prune topology is called. This step involves removing links with insufficient bandwidth, unidirectional connected links and links with missing properties. The step does not actually delete the links from the topology as the topology might be reused for later requests. Instead the link is marked as invalid, which also requires a very small amount of processing power compared to removing a link. This step is multi-threaded to speed up the step as well as improve the utilization of a multi-core CPU.

The next step is the most important part of the process, calculating path in the topology. Described in detail in the next section 3.6.3.

Depending on the configuration the next step will validate all the calculated paths. If the module is calculating something smaller than all the paths the validation done preformed during the actual path computation step, and this step will not be executed. The hypothesis behind this design decision is that it might be faster to validate all paths in parallel after, instead of validate in the single-threaded path computation step. This is only possible when calculating a large number of paths since a) all paths might not be valid, and new paths cannot be calculated after the path computation step, and b) only for a large number path will the speed benefit of parallelization overcome the cost of thread synchronization.

The last step in the process is to extract the ERO of the path, encode it and return the result to the RCE request handler.

The multithreading in the prune an validation steps in implemented using the Open Multi-Processing (OpenMP) API. The API provides a set of functions and $\mathrm{C}$ pragma directives to multi-thread blocks of $\mathrm{C} / \mathrm{C}++$ code [2]. The $\mathrm{C}$ pragma directive gives the compiler instructions on how and what blocks to run in parallel. And as it's implemented via pragma the directives will be ignored if the compiler lacks OpenMP support or if it's not enabled. 


\subsubsection{Path Computation Algorithm}

Input: Source/destination node, number of paths (kpaths)

Result: Computed Path, or error

1 validPaths $\leftarrow$ emptylist;

2 candidatePaths $\rightarrow$ enqueue (sourceNode);

3 while candidatePaths not empty do

4 currentPath $\leftarrow$ dequeueFirstPath (candidatePaths);

$5 \quad$ currentNode $\leftarrow$ lastNodeInPath (currentPath);

6 if currentNode.visited then

7 . continue

8 end

9 foreach Link currentLink in currentNode do

10 if resultsInLoop (CurrentLink) then

$11 \quad$ continue

12 end

13 if isVaild(currentPath, CurrentLink) then

14 continue

$15 \quad$ end

16 if currentLink equals destinationNode then

$17 \quad$ validPaths $\rightarrow$ append (currentPath, CurrentLink);

$18 \quad$ if length(validPaths) equals kpaths then

19

20

21

22

23

24

25

26 end

27 if validPaths not empty then

28 return validPaths

29 else

30 | return "Not path found"

31 end

Algorithm 1: Path computation algorithm used. Requires a source and destination node for input and returns a path or error message.

The algorithm takes tree arguments, the source and destination router and the number of routers to calculate. The function will return even either a) the number of paths requested has been calculated or b) when all possible paths has been evaluated. 


\section{- Lines 1-2: Initialization}

Set the valid paths to empty list, enqueue the source node in the candidatePath queue.

\section{- Line 3: Calculation loop}

Execute loop while there are still candidate paths left to evaluate.

\section{- Line 4-8: Evaluate node, examine the links}

Pop the first path from the queue, and set the last node of the path to currentPath. Optional optimization, check if the node has already been visited, if so skip the node. Iterate over all outgoing links for the node.

\section{- Line 9-15: Evaluate link}

Check if the current link will create a loop if appended with the path, if true then process next link. The next step is to check if the path appended with the link is still a valid path, i.e. creates a valid data plane path and meets the user's constraints, otherwise discard link and continue with the next link.

\section{- Line 16-23: Check the new path}

If the previous checks were successful the link can be appended to the current path. If the destination node is equal to the links remote end, then a path from source and destination has been found. Add the path to the calculated paths and check if the number of calculated path equals the number of requested path, if equal return the paths.

Lastly check if the remote end is not the destination node then add the path to the candidate paths.

\section{- Line 27-31: Return result}

If the list of the valid paths is empty no path from the source to the destination was found the algorithm returns an error message, else return the calculated paths.

Because of the algorithm uses a stack, which needs to be accessed in order, multithreading the algorithm is hard, but possible[11]. It's difficult to separate the processing of the candidate paths while still inserting and deleting path in the correct order. It would still be possible to perform the link processing (line 7-20) in parallel, but is unlikely to speed up the algorithm because of the thread synchronization cost except for node with a very large amount of links.

The loop detection function resultsInLoop used on line 7 , is optimized to use an path specific hash table containing the router ID of the routers in the path. To detect if this path could create a loop the algorithm simple checks if the router ID exists in the hash table. The complexity of the look up should be $\mathrm{O}(1)$ in the best case, and worst case $\mathrm{O}(\mathrm{n})$. Iterating each link and checking the router IDs would be an $\mathrm{O}(\mathrm{n})$ operation and therefore generally slower.

An optimization is done on line 6 of the algorithm, which forces PCE to only visit a node once, this reduces the computational time immensely (see section 4.4.1). But 


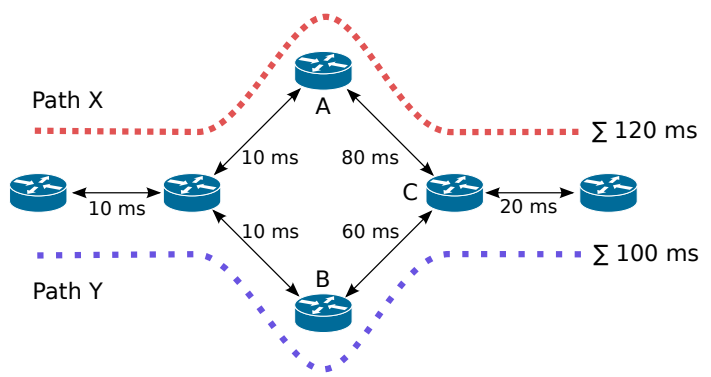

Figure 3.14: The network fragment contains two possible paths, path $\mathrm{X}$ and path $\mathrm{Y}$. The user has request a maximum latency of $110 \mathrm{~ms}$, this results in that only path $\mathrm{Y}$ is valid and path $\mathrm{X}$ is not. But when the optimization on line 6 of algorithm is enabled the PCE may fail to calculate the valid path Y.

this optimization may create problems for a small subset of validation constraints; the only such constraint implemented in this PCE is the cumulative constraints. Consider for example the network fragment in figure 3.14 where the user put a $110 \mathrm{~ms}$ latency constraint on the path. When the PCE is using the optimization it may not produce the path $\mathrm{Y}$, the path who meets the latency constraint. The reason is that when the $\mathrm{PCE}$ is evaluating the $\mathrm{X}$ path at router $\mathrm{C}$ it will mark that node as visited and path valid (as the path is currently under the $110 \mathrm{~ms}$ limit). In the next step the algorithm will evaluate the Y path at the same node (router C), but since the node is already visited it will discard the path. The problem arises in the next step when the algorithm goes to the last link and realizes that the $\mathrm{X}$ path is invalid, as the latency is over the limit. Because of this issue the optimization cannot be used when the user has specified a cumulative constraint.

\subsubsection{Path Validation}

Path validation involves checking if the current or fully calculated path meets the user constrains, for example bandwidth, and that the path constitutes a valid path in the data plane.

The implementation contains a function "isValid" which takes the current path and the next link and return true if the current path with the new path still constitutes a valid path. The function preforms the following checks:

\section{Layers}

Each layer transition is detected according to RFC4206 and each layer increase is stored in a stack [26, when a layer decrease is found the top stack item i retrieved and the two are compared. The switch capabilities and potential LSC properties of the two are compared, if they do not match the link is not valid. 


\section{LSC: User defined constraints}

Previous master thesis work in added LSC user defined constraints [28, this feature was also ported in to the path validation implementation. During each step in the path a accumulated set of user defined constraints are added. If the accumulated value exists the users maximum level the path is not valid.

\section{LSC: Path validation}

LSC path validation was added in a previous thesis and was incorporated in to the new path computation algorithm. Each LSC link incorporates a "Grid" object containing LSC properties, such as available wavelength and if a port is able to convert from one wavelength to another. This information is used to check if the new LSC link is compatible with the previous LSC link.

\section{Ethernet: Basic configuration validation}

Two separate checks are done to validate that the link and the switch transitions are valid. The first checks the link, the output from the remote interface is matched to the local interface input. If they do not match then the link is invalid, this check could also be done in the prune topology step to improve performance.

The second check if the input and output of the two interfaces on the switch. The previous links remote end output is check against the current links local end input. If they do not match the path is invalid. For example the previous links remote ends output maybe I-Tag but the current link local end requires a B-Tag as input, this is not a valid path as they do not match.

\section{Ethernet: Invalid layer hop}

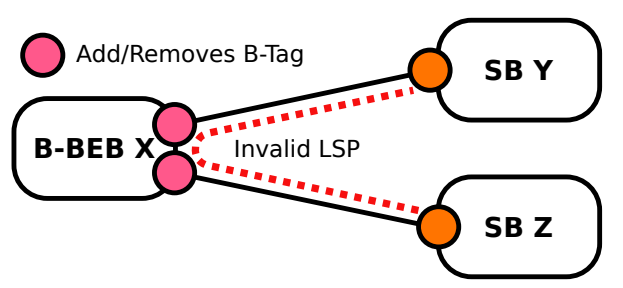

Figure 3.15: The LSP (marked in dotted red) is invalid, the tag would be added to the frame when entering and remove when exiting the router, and that layer would therefore only exist inside the router.

If a path contains an upper layer which is only used once, e.g. the layer only contains one router, this will create an invalid path. The reason is that an FA-LSP with only a single router cannot be created. Consider the network fragment in figure 3.15. The network consists of two SB-based switches connected to a B-BEB switch. A path going from $\mathrm{Y}$ to $\mathrm{Z}$ via $\mathrm{X}$ would require an layer increase between the 
two ports on the B-BEB. Since this layer would only exists inside the switch this cannot be signaled in the control plan. This specific example would still be possible to realize in the data plane, so this is a control plane limitation. A solution for this situation is discussed in the last part of section 3.3 .

\section{Ethernet: Layer validation}

A layer changes in ML Ethernet data plane is based on additions and removals of tags, and is encoded in the ISCD specific rules. To validate the layer transitions a table containing the type of tag and the value is created. Each time a tag is added the value for that tag is incremented. The opposite is done for tag removals, the value is decreased. This is done for each link, and when the last link in processed the table is checked. The value for each tag type is checked, and if the value in not zero the path contains unmatched layer changes and is therefore invalid.

\subsubsection{Virtual Topologies}

The path computation daemon RCE contained support to load a virtual topology, described in a configuration file. This feature was extended to support some missing pieces, such as unnumbered interfaces. This made it possible to use virtual topologies when evaluating the path computation implementation.

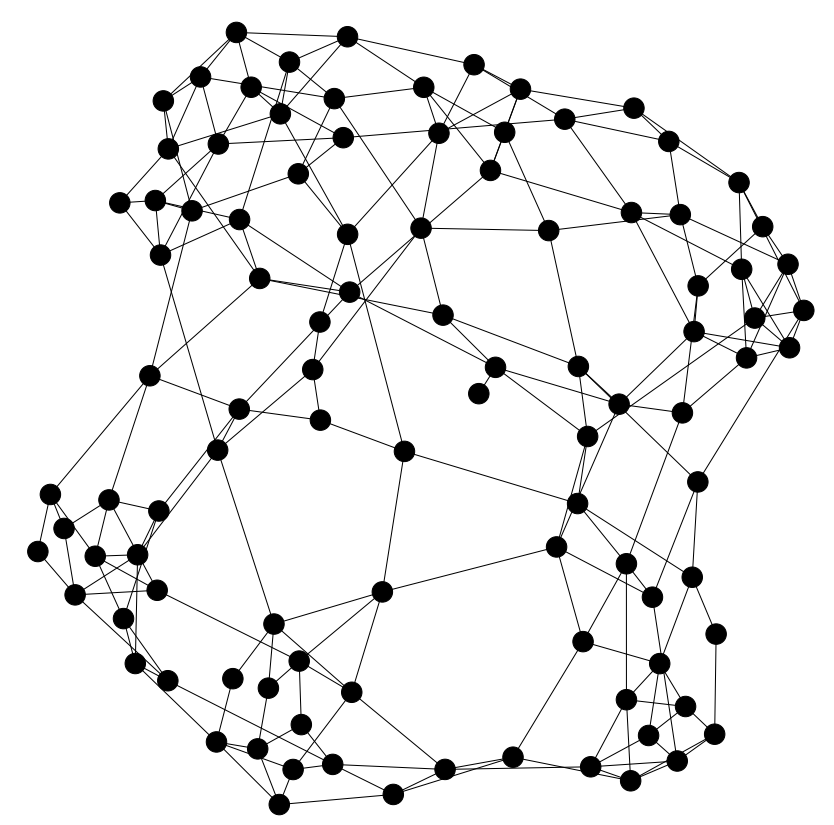

Figure 3.16: Visualization (using the application Gephi[1]) of a generated network, using the Waxman model, consisting of 100 nodes.

To construct the topology configuration files a Java application was developed. The application would generate a random network graph of desired size and output 
both an RCE topology configuration file and an DOT source file for network visualization.

Input: Number of nodes (count)

Result: Computed topology

1 probability $\leftarrow \beta$;

2 routers $\leftarrow$ emptylist;

3 foreach i in count do

$4 \quad$ routers $\rightarrow$ append (Router (nextAddress ()));

5 end

6 foreach router in routers do

\begin{tabular}{l|l}
7 & foreach otherRouter in routers do
\end{tabular}

\begin{tabular}{l|l|l}
8 & if router equals otherRouter then
\end{tabular}

$9 \quad \mid$ continue

$10 \quad$ end

11 if Random (probability) equals 0 then

$12 \quad$ if router $\rightarrow$ hasLinkTo(otherRouter) then

13

14

15

16

17

18 end

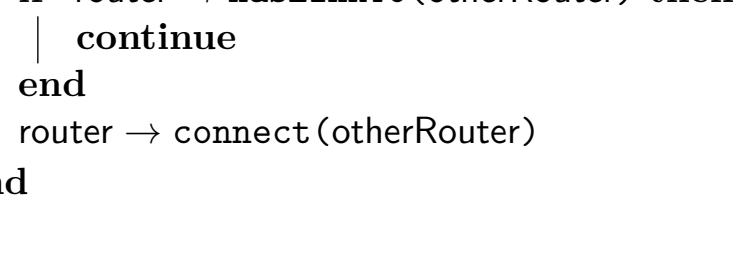

Algorithm 2: Erdős-Rényi algorithm

The first attempt to generate virtual topologies was done using the Erdős-Rényi model, specifically using the $\mathrm{G}(\mathrm{n}, \mathrm{p})$ variant. This was choose because that the model may creates "islands" of node when the right parameters, this effect can be used to create groups of router which then can be connected to create the full network. With the G(n, p) model a set of $n$ nodes are generated and each node is connected with every other node with a probability p. When np is smaller than one the generated graph will contain small clusters of node.

The implementation of algorithm first sets the probability (line 1 ) to $\beta$, which is calculated by count $+\frac{\text { count }}{7}=\frac{7 \cdot \text { count }}{6}$, the resulting probability to connect a node with any other node will be $\frac{\text { count }}{\frac{7 \cdot \operatorname{count}}{6}}=\frac{6 \cdot \text { count }}{7 \cdot \text { count }}=\frac{6}{7} \approx 0.8571$ which is below the desired zero. The next step is to create all the routers and initialize them with a unique IP address (lines 3-5).

The main calculation is done on line 6-18 were each link is iterated. For each iteration the list of router are again iterated. In the inner loop the first check will make sure that the implementation does not create links to the router itself. The next step is to call the pseudo random generator which will return a value a value between 0 and probability. If the returned value is zero the two routers will be connected if not already connected. 
Unfortunately the Erdős-Reńyi model does not take the distance between to nodes in to account, and therefore generates long links between nodes. This creates a problem when using the generated topologies for benchmarking a path computation implementation as the paths will be short, even for large network. This is also not realistic as it would be very costly to lay down very long links, especially when there are an existing path between the two node that could be used.

Because of the issue another implementation was constructed, based on the Waxman model[38]. With this model two node are connected based on a probability derived from the distance between the nodes, in contrast to the Erdös-Reńyi model where the probability is fixed. Two node who are close will therefore be connected with a higher probability compared to two nodes further apart.

The probability is given by the function $\beta \exp \left(\frac{-\operatorname{distance}(u, v)}{\operatorname{maxDistance} \cdot \alpha}\right)$, where $\mathrm{u}$ and $\mathrm{v}$ are nodes and maxDistance the maximum distance possible. A small value for the constant $\alpha$ will produce a graph with shorter links while large value of $\beta$ increases the number of links. 
Input: Number of nodes (count)

Result: Computed topology

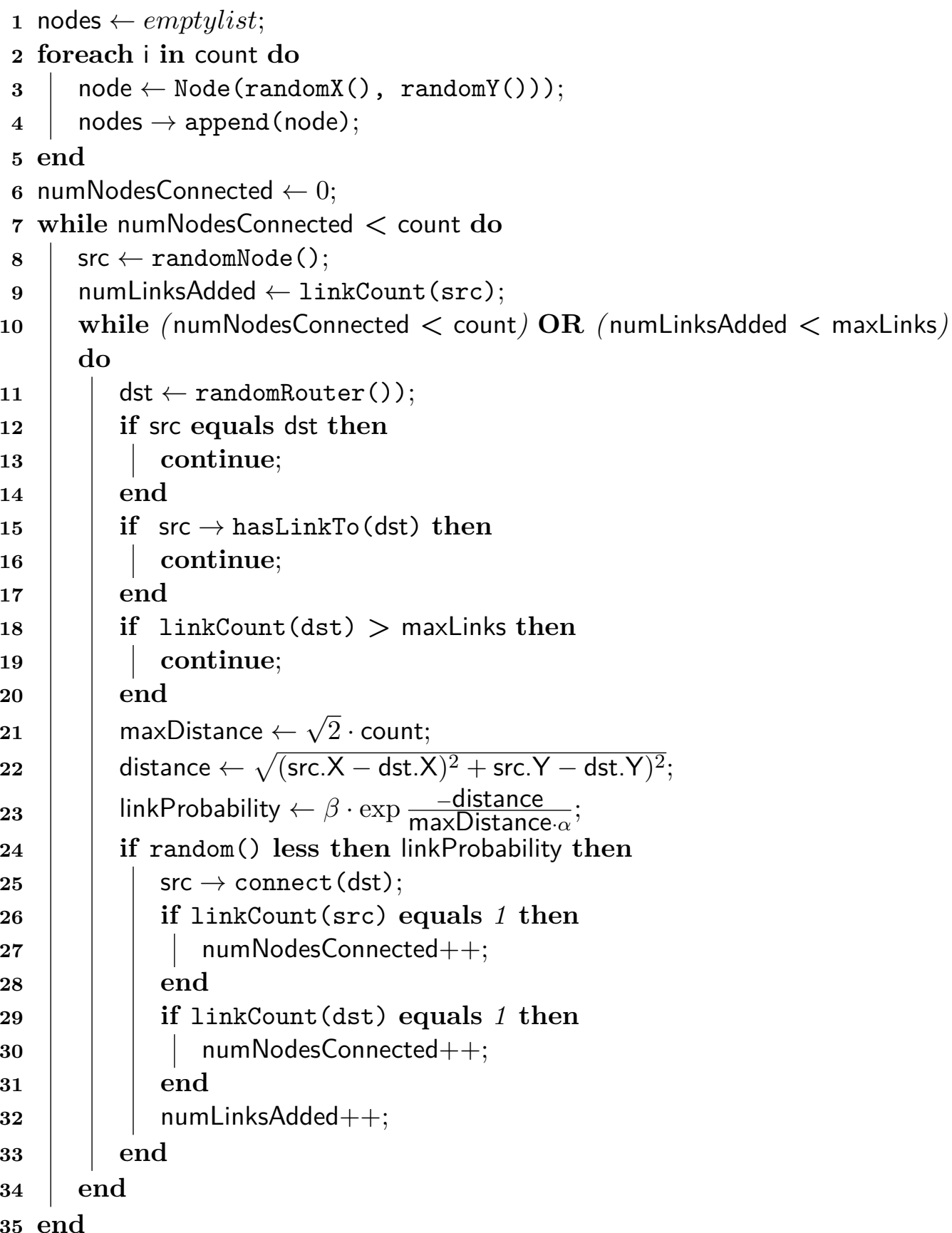

Algorithm 3: Waxman based topology generator

This implementation was based the implementation from the BRITE project [9], and takes four different parameters, the number of routers, the maximum links per 
router and the $\beta$ and $\alpha$ constants and works as follows:

\section{- Lines 1-6: Initialization}

The number of requested node are created and assigned a random $\mathrm{X}$ and $\mathrm{Y}$ coordinate. It will Also check so two nodes are not placed in the same position.

\section{- Lines 7-10: Main loop}

While the number of connected nodes are less than all nodes, pick a random router from the list of routers and fetch the number of links that node has. Try to connect this router to another router while the number of connected nodes are less then the total number of nodes and so long as the number of links from/to this node is less the maximum allowed.

\section{- Lines 11-20: Check remote node}

Fetch a random router and check so the two nodes are not the same. Then processed by checking if the two nodes are already connected and if the other node already has the maximum number of links, if so start from the beginning.

\section{- Lines 21-33: Try to connect}

Calculate the link probability using the Waxman formula. Then generate a random value and if this value is less than the probability a connection should be made. Connect the two nodes and see if this connection was the first connection for either one, if so increase the number of connected nodes.

When the graph is generated the implementation will check if any network partitions exists. This is done by simply waking through the graph and mark each visited node. When no more nodes can be reached the number of nodes visited are compared to the total number of nodes. A network partition exists if the number of reached nodes are smaller than the total number of nodes.

\subsection{PCE-NMS integration}

The current implementation of the PCE implements a TED which only collects information from OSPF-TE. While this works well for networks with small number of nodes this approach might introduce scaling problems as the number of nodes and interfaces increases.

Previous work has mostly focused on a pure NMS backed TED with no data provided by OSPF [36, 35, 31]. While this approach will mitigate the problem of OSPF scaling, it might introduce an considerable amount of overhead depending on the implementation. For example if this is done by polling each network element.

The information contained could be categorized as static information, e.g. hardware properties, and parameters not likely to change, and dynamic information, i.e. usage depended properties and configurable properties.

From table 3.2 it can be seen that the most frequent information are the bandwidth on links, these values and other frequently updating values would be ideal to 


\begin{tabular}{|l|l|}
\hline Property & Update frequency \\
\hline \hline Number of interfaces & Static \\
\hline Requires device power of to change \\
\hline Link speed of interface & Very rare \\
\hline Physical change to topology or deliberate configuration modification. \\
\hline Interface switch capability/encoding & Very rare \\
\hline Either static or configured on deployment. \\
\hline Interface port type reconfiguration & Rare \\
\hline Either static or configured on deployment. \\
\hline Maximum Bandwidth \\
\hline Either static or configured on deployment. \\
\hline Max Reservable bandwidth & Very rare \\
\hline Changes on new LSP setup. & Frequent \\
\hline ISCD specific label usage & Frequent \\
\hline May changes on new LSP setup.
\end{tabular}

Table 3.2: The update frequency of different values distributed by OSPF-TE.

have in OSPF. The other values could be acquired from a NMS instead. This should decrease bandwidth usage and convergence time of OSPF, and should increase the scalability of the OSPF area.

If the NMS is MTOSI enabled the PCE could fetch the status of the network using the getAllEquipment (which returns all the network equipment) operation iteratively. Information for a specific element could be fetch more frequently using the getEquipment operation.

The information returned from the aforementioned operations could be parsed and converted from MTOSI model to a OSPF-TE model and inserted in to the existing OSPF-TE based TED. Some filtering of the data would be required as the MTOSI model allows for modeling several termination points over each other, and some termination point would therefore be unnecessary to have in the TED. The concept similar to GMPLS switching capabilities exists in MTOSI in the name of "Layer rates" 24], these values could be used to map a termination point to a GMPLS layer. Possible mapping between these values and GMPLS switch capability can be seen the table 3.3 .

The mapping between the MTOSI network elements and the OSPF-TE information is more complicated and care should be taken when the MTOSI model of the network is created to make the mapping easier. For example MTOSI naming of managed elements are not required to be address of the element and could therefore be an arbitrary name 25. To make the mapping easier this name would be set to the IP address of the router. Another solution would be to have a look up table between the name in MTOSI and the network address of the element.

The situation is similarly in the naming of termination points, there the name 


\begin{tabular}{|l|l|l|}
\hline MTOSI Layer & MTOSI Layer Technology & GMPLS Switch Capability \\
\hline \hline LR_DSR_10Gigabit_Ethernet & 10 Gbit/s Ethernet & L2SC/ML Ethernet \\
\hline LR_DSR_Fast_Ethernet & $10 / 100$ Mbit/s Ethernet & L2SC/ML Ethernet \\
\hline LR_Ethernet & All Ethernet rates & L2SC/ML Ethernet \\
\hline LR_MPLS & MPLS & PSC \\
\hline LR_Optical_Channel & WDM wavelength (frequency) & LSC \\
\hline LR_Optical_Multiplex_Section & WDM wavelength bands (oms) & LSC \\
\hline LR_Optical_Transmission_Section & WDM entire optical signal & LSC \\
\hline LR_OPTICAL_SECTION & Single-lambda ports & LSC \\
\hline LR_T*_and_DS*_*M & PDH & TDM \\
\hline LR_VT*_and_TU*_VC* & SONET/SD, path signal & TDM \\
\hline LR_Line_OC*_STS*_and_MS_STM* & SONET/SD, STM multiplex section & TDM \\
\hline LR_STS*_and_VC3_* & SONET/SD, Contiguous Concatenation & TDM \\
\hline LR_PHYSICAL_OPTICAL & Signal on optical physical media & FSC \\
\hline
\end{tabular}

Table 3.3: MTOSI Layers and possible mapping to GMPLS Switch capabilities. A asterisk indicates a numerical value.

value could for example be "wdm:1:4:3-4", which includes the frequency and port identifier but the naming it somewhat arbitrary. This value could be set to the GMPLS interface identifier which should make it easier to map the termination point to the OSPF-TE values.

MTOSI is a very large standard and allows for many different scenarios and the work in this thesis only scratches the surface. More work on the mapping and implementation is needed.

\subsubsection{MTOSI for a ML Ethernet}

The MTOSI standard contains several useful parameters related to the Ethernet layer, contained in the layer parameters. The current ISCD specific information in OSPF-TE does not contain information on the currently in use VID/I-SIDs, nor there mapping (when using label swapping). This was done to limit the amount of information disseminated through the OSPF network as the VID/ISID data can grow to a large size.

MTOSI already contains the necessary field for collecting the CVID and SVID usage, and the potential mapping between the values. Unfortunately the current standard does not include fields for the 802.1AH standard (e.g. ISID and BVID), and needs extended to support those.

In the layer parameters for the Ethernet layer contains a set of "Tag Translation" field used to encode tag mapping, one definition for CVIDs and one for SVIDs. The definition contains three fields, "TagTranslation_Table_External" and "TagTranslation_Table_Internal" contains the external to internal VID mapping. And the field "TagTranslation_Table_Count" contains the number of tags in the table. 


\begin{tabular}{|c|c|c|}
\hline MTOSI Field & Abbreviation & Possible OSPF mapping \\
\hline Management Domain & $\mathrm{MD}$ & OSPF Area \\
\hline \multicolumn{3}{|l|}{ String identifying a domain. } \\
\hline Managed Element & $\mathrm{ME}$ & Router ID \\
\hline \multicolumn{3}{|c|}{ IP address of an network element } \\
\hline Physical Termination Point & PTP & Physical Interface \\
\hline \multicolumn{3}{|c|}{ String with a interface name, and additional parameters. } \\
\hline Floating Termination Point & FTP & Abstract (internal) interface in an abstract link, IACD \\
\hline \multicolumn{3}{|c|}{ String with a interface name, and additional parameters. } \\
\hline Connection Termination Point & $\mathrm{CTP}$ & TE Link interface, FA-LSP advertised as TE-Link \\
\hline \multicolumn{3}{|c|}{ String with a interface name, and additional parameters. } \\
\hline Topological Link & TL & A pair of interfaces, with additional with interface parameters. \\
\hline \multicolumn{3}{|c|}{ A list of Termination points, direction of the link, and layer information. } \\
\hline Protection Group & $\mathrm{PG}$ & PG Link Protection Type, Shared Risk Link Group \\
\hline
\end{tabular}

Table 3.4: MTOSI fields and possible mapping to OSPF-TE attributes.

These field can be used to store the used VID by setting the VID values to be equal in both table, even if translation is not used.

The MTOSI Ethernet parameters also contains other useful parameters, the "PVID" parameter is use to indicate which VID value will be used for tagging untagged frames. This could be used when configuring port based LSPs. 


\section{Chapter 4}

\section{Verification and analysis}

To verify the design and implementation of the ML Ethernet solution two ML Ethernet network testbeds were constructed to functionally test the solution. The design of the control plane was tested to provide information on how the label and switching capability design preformed in a real and functional network. The setup and forwarding aspects of the data plane were also tested using the testbeds, for example how well the data plane design handled FA-LSP. The primary network consisted of a large pure multi-layer network, and the secondary network consisted of a smaller number of nodes together with a few none ML Ethernet nodes. The smaller network was constructed to demonstrate end-to-end service allocation between the home network and the access network.

The PCE was also benchmarked to provide information on how the implementation scaled, important as an access network may contain a large number of nodes. This was accomplished by using larger virtual topologies, as such large networks would be costly to implement in a testbed solution.

\subsection{Testbed}

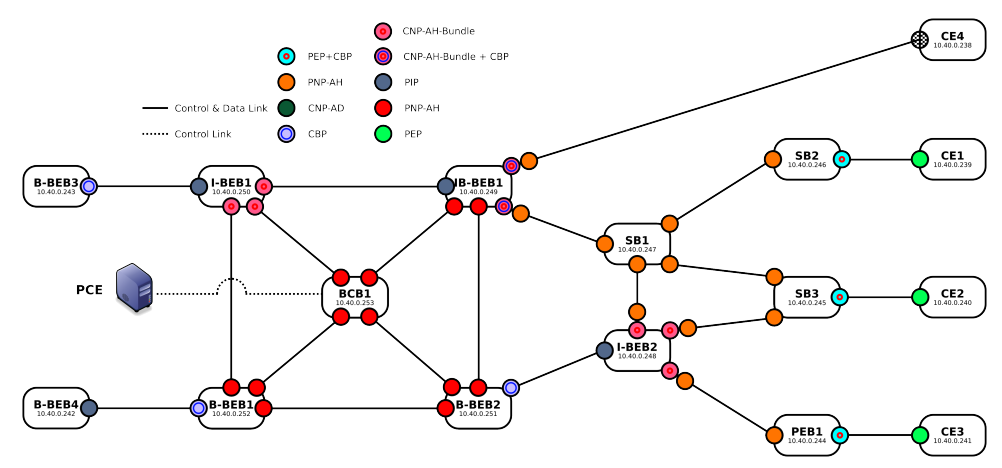

Figure 4.1: The Ethheir testbed configuration 
The primary testbed consisted of sixteen virtual machines running Ubuntu 9.10. One additional virtual machine was running the PCE software and was connected to network on the control plane level only. The host machine was a Dell server with dual Intel Xeon E5520 CPUs; each CPU contained four hyper-threading enabled $\mathrm{CPU}$ cores running at $2.27 \mathrm{GHz}$. The virtual machines where hosted using KVM and configured with one CPU core and 250MB of memory. The PCE was running on separate but identical host machine, with $8 \mathrm{CPU}$ cores and $4 \mathrm{~GB}$ of ram configured. Each machine was connected to a common Linux kernel based bridge used for control plane traffic. The control plane links were implemented via GRE tunnels. Data plane connections where implemented as pair of interfaces connected via a kernel based bridge.

The network layout was based on the sample PBB network described in IEEE 802.1AH standard. Five routers (BCB1, I-BEB1, IB-BEB1, B-BEB1 and B-BEB2) constitute the "backbone" network and switch on the B-tag. Connected to the core network are the border routers (IB-BEB1, I-BBE2 and I-BEB1) who provide I-SID encapsulation. The S-tag capable switches (SB1-3 and PEB1) are directly or indirectly connected to the I-tag capable switches. The C-tag enabled switches (CE1-4) are connected to S-tag capable switches and represent can represent either access switches or client switches.

\subsection{Demonstration network}

A smaller multi-layer Ethernet network was created as part of a larger network to demonstrate cross-border end-to-end resource allocations. The demonstration setup included an UPnP-QoS network[4], an UPnP-QoS and GMPLS aware gateway and a ML Ethernet based access network.

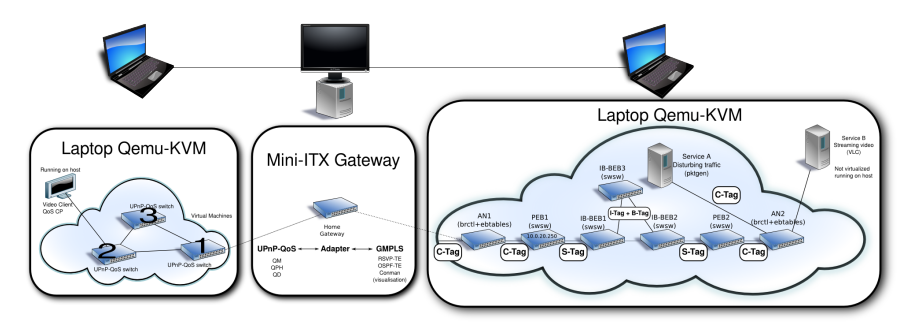

Figure 4.2: Network setup used to demonstrate end-to-end allocation of QoS service in a access network.

The access network consisted of seven routers, with two C-tag based edge nodes, two nodes with S-tag capability and tree core nodes capable of handling both I-tags and B-tags. The core nodes were arranged in a triangle with one of the links having a smaller amount of bandwidth. This was done do to illustrate that the PCE calculates a different path for high bandwidth requests. 
The edge node were not implemented using the SWSW based data plane, instead they used a older implementation previously developed at Acreo[34. The old data plane used the Linux bridge functionality [33] (C-tag only) with a different RSVPD data plane controller. The reason for using this older solution was that the SWSW switch was not compatible with the Linux traffic shaping functionality.

\subsection{Functional Testing}

Using the testbed several LSP created from and to the CE nodes in the network. The LSPs was configured with different bandwidth and VID values. When committing the LSP the resulting FA-LSP was observed on the layer borders.

Virtual tagged interfaces were created on both sides of the LSP to map traffic into the correct LSP. This was done using Linux vconfig commands. To provide IP connectivity each side was configured with an IP address and a static ARP entry was added for the IP destination. This is necessary as the LSP is using the VID+MAC combination for label, and therefore will not forward Ethernet broadcast traffic which is needed for ARP request.

Using the IP connectivity of LSP the implementation was tested by running application and benchmarking tools, such as the "iperf" IP benchmarking tool[5]. The traffic generated was investigated at all the nodes of LSP using the console version of the popular "wireshark" tool[8]. The implementation was verified by checking that the correct LSP payload reached the destination and that the correct Ethernet headers and value were present.

In the demonstration network the implementation was used to demonstrate endto-end quality of service (QoS) resource allocation. In the demonstration network the implementation successful established LSP which then were used to transmit video in. This setup was used during the ECOC 2010 Exhibition and for internal demonstration at Acreo.

\subsection{PCE Performance Evaluation}

The PCE implementation was tested both in the time aspect, i.e. how fast it was, and the memory efficiency. This was done to provide information on how the well the PCE scaled and on how fast the PCE could return a path. This is important as an access network may be large and setup time is important to provide a good end user experience.

\subsubsection{Time efficiency}

To measure the time efficiency of the PCE several large topologies was generated. The testbed network was not used as that topology in the TED has it has a very limited number of nodes and therefore would not provide any information about the scalability of the implementation. In addition path requests in such a small network 
would be calculated in a very small amount of time, probably small enough for the network latency to play a at lease as large part of resulting response time in real production use.

Five different virtual topologies was generated using the method described in section 3.6.4. Each topology had an increasing amount of routers, 2, 4, 6, 8 and 10 thousand. The topologies where flat, i.e contained not layer increases, the reason was partly that such topologies are harder to generate and more importantly such a network should not change the characteristics of the results. The reason for this is the checks need when calculating i a multi-layer network only adds a fixed amount of extra time per node hop, probably in the order of an extra $20 \%$ in time. It may also be that such a network would be faster to calculate in as more potential paths could be discarded during path computation.

For each topology a script was generated which contained two thousand request from random source to random destination. Before the real benchmark was started the first 100 request from the script was executed to provide warm up period, this is necessary as the network graph will be constructed on the first request. It will also allocate the base level of memory and other resources. After the warm up period the script was executed. The time until the client received a response was collected and stored in a resulting log file. This time is therefore the time it took the PCE to calculate the path, plus a very small amount of network overhead.

Two different variations of the PCE implementation were benchmarked, a single threaded version and a multi-threaded version. Both variations were configured with the optimization, discussed in the section 3.6.2, and set to calculate the best path in terms of path length. 


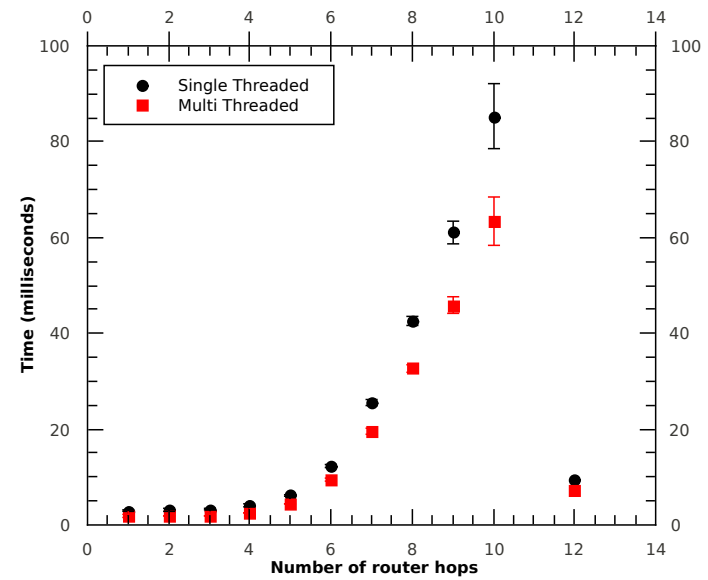

(a) Mean service time, 2k Routers, 4729 Links

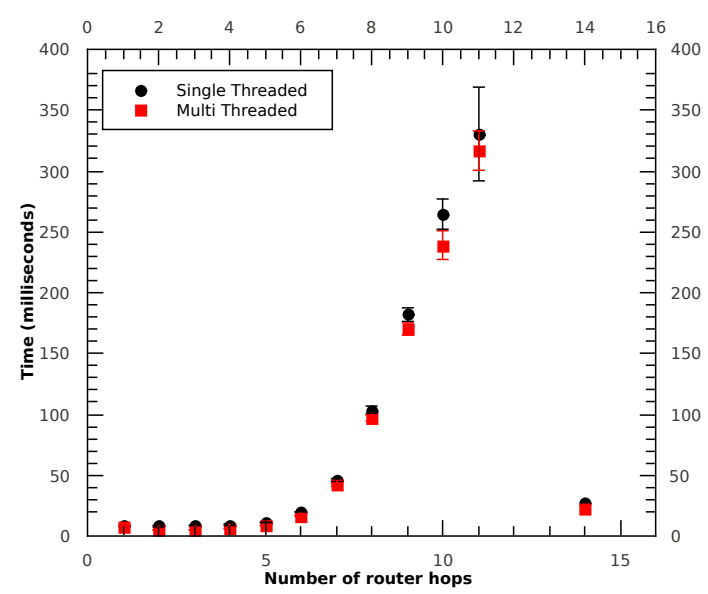

(c) Mean service time, 6k Routers, 14278 Links

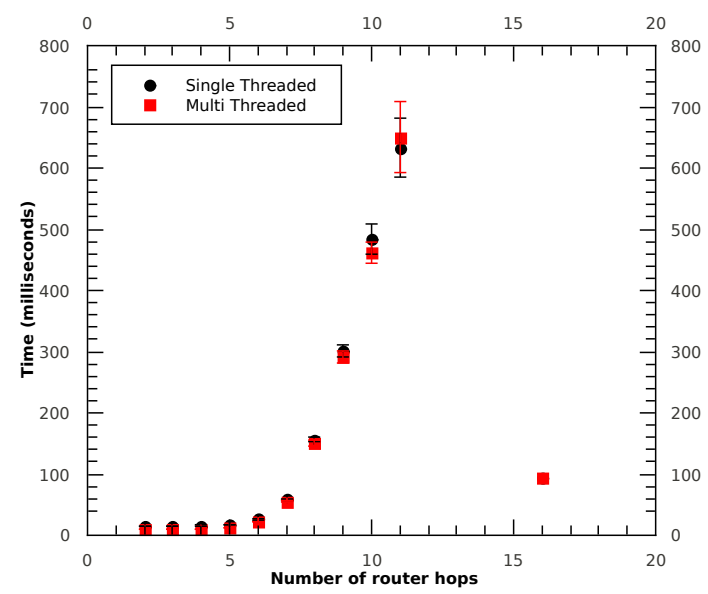

(e) Mean service time, 10k Routers, 23801 Links

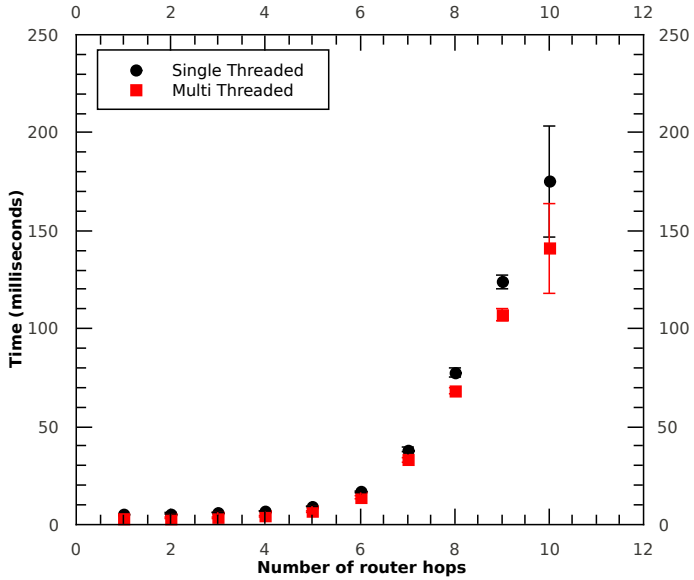

(b) Mean service time, 4k Routers, 9529 Links

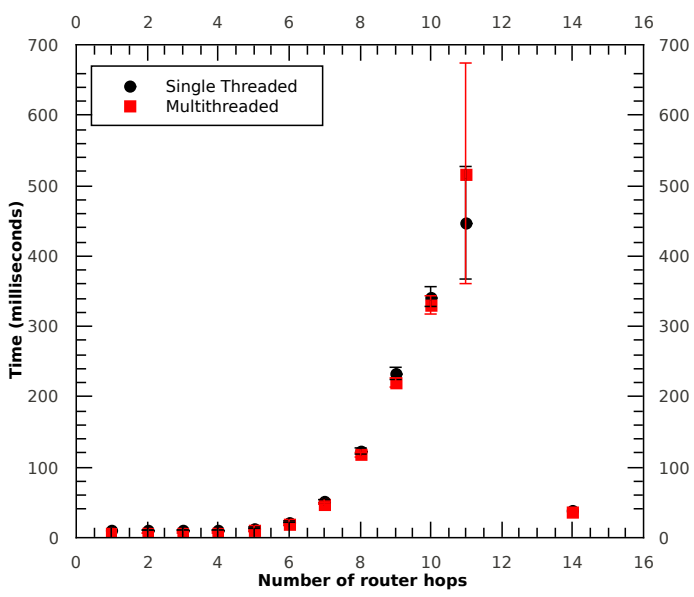

(d) Mean service time, 8k Routers, 18909 Links

Figure 4.3: Mean service time, e.g. time until the client receives a response, grouped by path length. 
The result showed that the time needed was growing exponentially, which is not surprising as the number of possible paths would grow in a similar fashion when the number of hops increases. There were a few data point who diverged from this, for example in figure 4.3c, a path resulting in 12 hops took a small amount of time to calculate. These anomalies are probably the result of a simple path in the topology, the number of possible between the two nodes where low.

When comparing the different sizes of the topologies a clear liner pattern can be seen between the average time to calculate a path in a network and the size of the network. Furthermore the average time for calculating path in such a large networks were low, depending on the desired time limit a very large network could still only use a single PCE. This could for example be the case when using GMPLS in a access network, where the number of end nodes might be large.
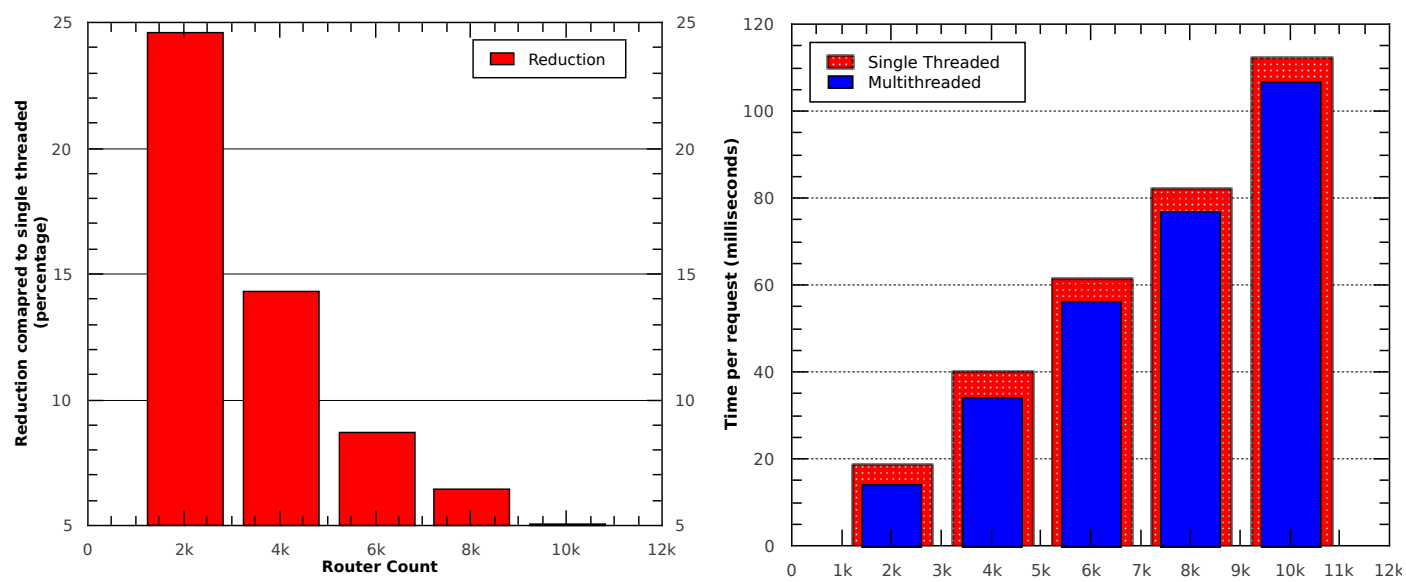

(a) The speedup using multithreading compared to(b) The mean time to calculate one path in each single threading. graph.

Figure 4.4: Graphs showing total value for each graph size.

The performance difference between the multithreaded and single threaded versions of the PCE was expected, the performance benefit decreased as the size of topology increases. The reason is that only the prune topology step was executed in parallel, and also constant for the topology, and the relative time reduction gained in this step therefore deceases as the time spent in the path computation step increases. 


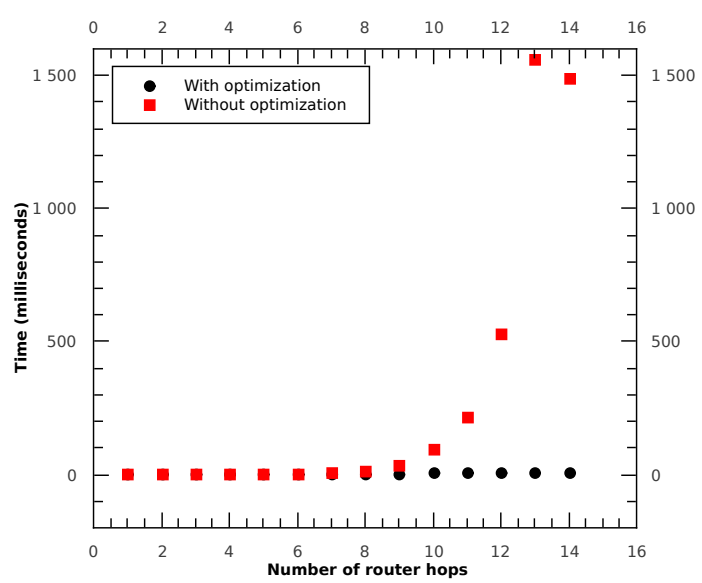

Figure 4.5: Graph showing two thousand request over a network with four hundred routers, both with the optimization and without.

The performance benefit of the optimization discussed in the section 3.6 .2 was tested using a topology with 400 nodes. The testing procedure was the same as for the benchmarks above. The result showed a immense performance improvement for longer paths, in total the optimized version was 15 times faster than the nonoptimized version. This optimization is therefore crucial when the topology is large.

\subsubsection{Memory efficiency}

The memory usage and characteristics was measured using the massif tool from the open source project valgrind[7]. The tool executes the RCE implementation and intercepts each request for memory and the amount of memory and the requesting component is stored. At the end of the execution the tool outputs a file which can be analyzed.

The virtual topology with 750 routers was used as backing to TED and 500 requests was made to the PCE. The data from massif tool was then used to produce the figure 4.6. Each spike in the figure present the increase memory usage during the path computation, with a especially demanding path near the end of the execution.

The results show that the implementation does not leak any considerable amounts of memory as none of the fields shows an constant increase. This also apparent as the memory usage goes down to a baseline usage after each completed request. This was further verified by running the memory problem detection tool in valgrind on the implementation. The result showed that there was no memory leaks in the path computation module.

It can be observed from the figure that most heap memory was consumed by the path computation process and the TED which was expected. In conclusion the heap usage of the implementation must be considered acceptable with a peak usage of $16.3 \mathrm{MiB}$ when computing on graph with 750 nodes.

Stack memory usage of the implementation is harder to acquire and was not 


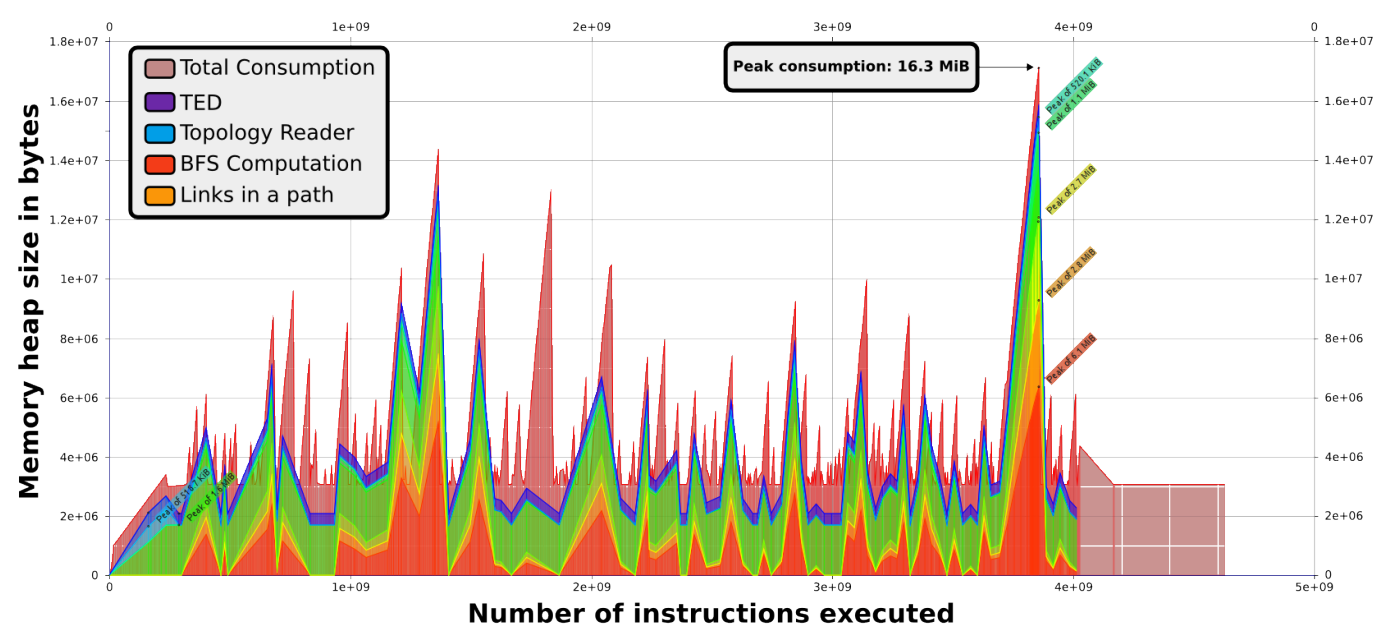

Figure 4.6: Visualization of RCEs heap memory usage while computing 500 requests.

done. But as the implementation is not using function based recursion, i.e. a function which calls its self, the stack memory usage should be stable and stack overflows are unlikely. 


\section{Chapter 5}

\section{Conclusions and future work}

The work in this thesis shows that a multi-layer hierarchical Ethernet network is possible and could create a use full data plane. While the design of the interface switch capabilities works well, it does not fully flow in the design spirit of other GMPLS data planes. This is most evident in that the layer in it is self also contains layers, even if TDM has a similar situation (where the timeslots can have sub-timeslots) the layers in ML Ethernet is more physical and therefore different. An implementation using different switch capabilities, one for each tag type, would be interesting to investigate. It might be possible by using a combination of ISCD, IACD and some restrictions of the combinations of network element configurations. Point to multipoint LSP was not considered and would also be interesting to investigate.

The PCE implementation showed very good performance when using the optimization discussed in the section 3.6.2. The performance was good enough to use the PCE in even very large network. It should be noted however that the PCE could not compute disjoint path, used for implementing protection, which would be useful even in an access network.

The code implementation did not fully implement the port based LSP functionality and this need to be further explored and implemented. Also the ability of use one-to-one mapping of S-VID values to I-SID value was not fully explored; this presents an interesting problem as a label would be converted to and from a different layer label.

The integration of MTOSI information and OSPF-TE information in to the TED needs more work. The mapping between MTOSI and OSPF-TE is incomplete and needs to be investigated further. A code implementation which is able to utilize information from both sources, would be desirable as this could provide more information about potential issues and may provide information on possible scalability and convergence improvements.

In conclusion the thesis showed that a multi-layer Ethernet solution presents a promising new data plane in GMPLS. 


\section{Bibliography}

[1] Gephi, an open source graph visualization and manipulation software, 2008. URL http://gephi.org/.

[2] OpenMP application program interface version 3.0, 2008. URL http://www . openmp.org/mp-documents/spec30.pdf.

[3] Understanding pbb-te for carrier ethernet, 2008. URL http://www.fujitsu. com/downloads/TEL/fnc/whitepapers/UnderstandingPBBTE.pdf.

[4] Upnp-qos architecture, version 3.0, 2008. URL http://www . upnp.org/specs/ qos/UPnP-qos-Architecture-v3.pdf.

[5] Iperf, 2010. URL http://iperf . sourceforge.net/.

[6] Path computation element protocol (pcep) numbers, 2010. URL http://www. iana.org/assignments/pcep/pcep.txt.

[7] Valgrind, 2010. URL http://valgrind.org/

[8] Wireshark, 2010. URL http://www.wireshark.org/.

[9] Ibrahim Matta John Byers Alberto Medina, Anukool Lakhina. Brite: Boston university representative internet topology generator, 2002. URL http://www . cs.bu.edu/brite/.

[10] D. Awduche, L. Berger, D. Gan, T. Li, V. Srinivasan, and G. Swallow. RSVPTE: Extensions to RSVP for LSP Tunnels. RFC 3209 (Proposed Standard), December 2001. URL http://www. ietf.org/rfc/rfc3209.txt. Updated by RFCs 3936, 4420, 4874, 5151, 5420, 5711.

[11] D.A. Bader and K. Madduri. Designing multithreaded algorithms for breadthfirst search and st-connectivity on the cray mta-2. pages $523-530$, aug. 2006.

[12] L. Berger, D. Gan, G. Swallow, P. Pan, F. Tommasi, and S. Molendini. RSVP Refresh Overhead Reduction Extensions. RFC 2961 (Proposed Standard), April 2001. URL http://www.ietf.org/rfc/rfc2961.txt. Updated by RFC 5063 . 
[13] R. Braden, L. Zhang, S. Berson, S. Herzog, and S. Jamin. Resource ReSerVation Protocol (RSVP) - Version 1 Functional Specification. RFC 2205 (Proposed Standard), September 1997. URL http://www.ietf.org/rfc/ rfc2205.txt. Updated by RFCs 2750, 3936, 4495, 5946.

[14] R. Coltun. The OSPF Opaque LSA Option. RFC 2370 (Proposed Standard), July 1998. URL http://www. ietf.org/rfc/rfc2370.txt. Obsoleted by RFC 5250, updated by RFC 3630.

[15] Nabil Bitar Attila Takacs Don Fedyk, Himanshu Shah. Generalized multiprotocol label switching (gmpls) control of ethernet provider backbone traffic engineering (pbb-te), 2010. URL http://tools.ietf.org/id/ draft-ietf-ccamp-gmpls-ethernet-pbb-te-06.txt.

[16] Adrian Farrel. The Internet and Its Protocols: A Comparative Approach. Morgan Kaufman, 2004. ISBN 1-55860-913-X.

[17] IEEE. Std 802.1ad-2005, virtual bridged local area networks, amendment 4: Provider bridges, 2005.

[18] IEEE. Std 802.1q-2005, virtual bridged local area networks, 2005.

[19] IEEE. Ieee std 802.1ag-2007, virtual bridged local area networks, amendment 5: Connectivity fault management, 2007.

[20] IEEE. Std 802.1ah-2008, virtual bridged local area networks, amendment 7: Provider backbone bridges, 2008.

[21] IEEE. Std 802.1qay-2009, virtual bridged local area networks, amendment 10: Provider backbone bridge traffic engineering, 2009.

[22] Dr. Bijan Jabbari et al. Jerry Sobieski, Tom Lehman. Dragon project, 2010. URL https://dragon.maxgigapop.net/twiki/bin/view/DRAGON/WebHome

[23] D. Katz, K. Kompella, and D. Yeung. Traffic Engineering (TE) Extensions to OSPF Version 2. RFC 3630 (Proposed Standard), September 2003. URL http://www.ietf.org/rfc/rfc3630.txt. Updated by RFCs 4203, 5786.

[24] et al. Keith Dorking. GD1-17 layerrates, version 3.3, 2007.

[25] et al. Keith Dorking. SD1-25 mtnm support for a naming convention, version $3.4,2008$.

[26] K. Kompella and Y. Rekhter. Label Switched Paths (LSP) Hierarchy with Generalized Multi-Protocol Label Switching (GMPLS) Traffic Engineering (TE). RFC 4206 (Proposed Standard), October 2005. URL http://www.ietf.org/ rfc/rfc4206.txt. Updated by RFC 6001 .

[27] et al. Kunihiro Ishiguro. Gnu zebra, 2003. URL http://www.zebra.org/. 
[28] Alexander Lindström. GMPLS multi-layer networking:routing and constraintbased path computation sin optical network segments, 2007.

[29] Ju Liu. Analysis and implementation of a constrained path computation algorithm in a multi-layer GMPLS network, 2009.

[30] J. Moy. OSPF Version 2. RFC 2328 (Standard), April 1998. URL http: //www.ietf.org/rfc/rfc2328.txt. Updated by RFC 5709 .

[31] T. Otani. All-optical networking testbed demonstration. pages 1-2, aug. 2008.

[32] D. Papadimitriou and A. Farrel. Generalized MPLS (GMPLS) RSVP-TE Signaling Extensions in Support of Calls. RFC 4974 (Proposed Standard), August 2007. URL http://www.ietf.org/rfc/rfc4974.txt. Updated by RFC 6001.

[33] Jim Robson. Kernel korner - linux as an ethernet bridge. Linux Journal, 2005.

[34] Pontus Sköldström. Multi-region gmpls control and data plane integration, 2008.

[35] T. Tsuritani. Gmpls-controlled all-optical mesh networking demonstration using path computation-capable nms. pages $1-1$, sep. 2008.

[36] T. Tsuritani, M. Miyazawa, S. Kashihara, and T. Otani. Optical path computation element interworking with network management system for transparent mesh networks. pages $1-10$, feb. 2008.

[37] JP. Vasseur and JL. Le Roux. Path Computation Element (PCE) Communication Protocol (PCEP). RFC 5440 (Proposed Standard), March 2009. URL http://www.ietf.org/rfc/rfc5440.txt

[38] B.M. Waxman. Routing of multipoint connections. Selected Areas in Communications, IEEE Journal on, 6(9):1617 -1622, dec. 1988. ISSN 0733-8716. 
Appendices 


\section{Appendix A}

Graph Attributes

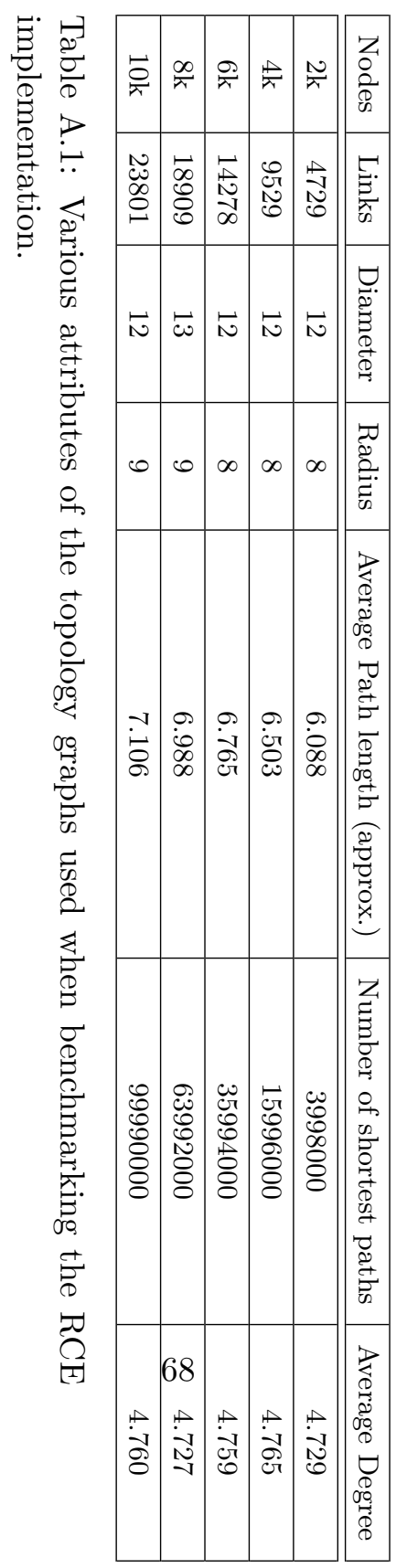




\section{Appendix B}

\section{Ethernet data plane overview}

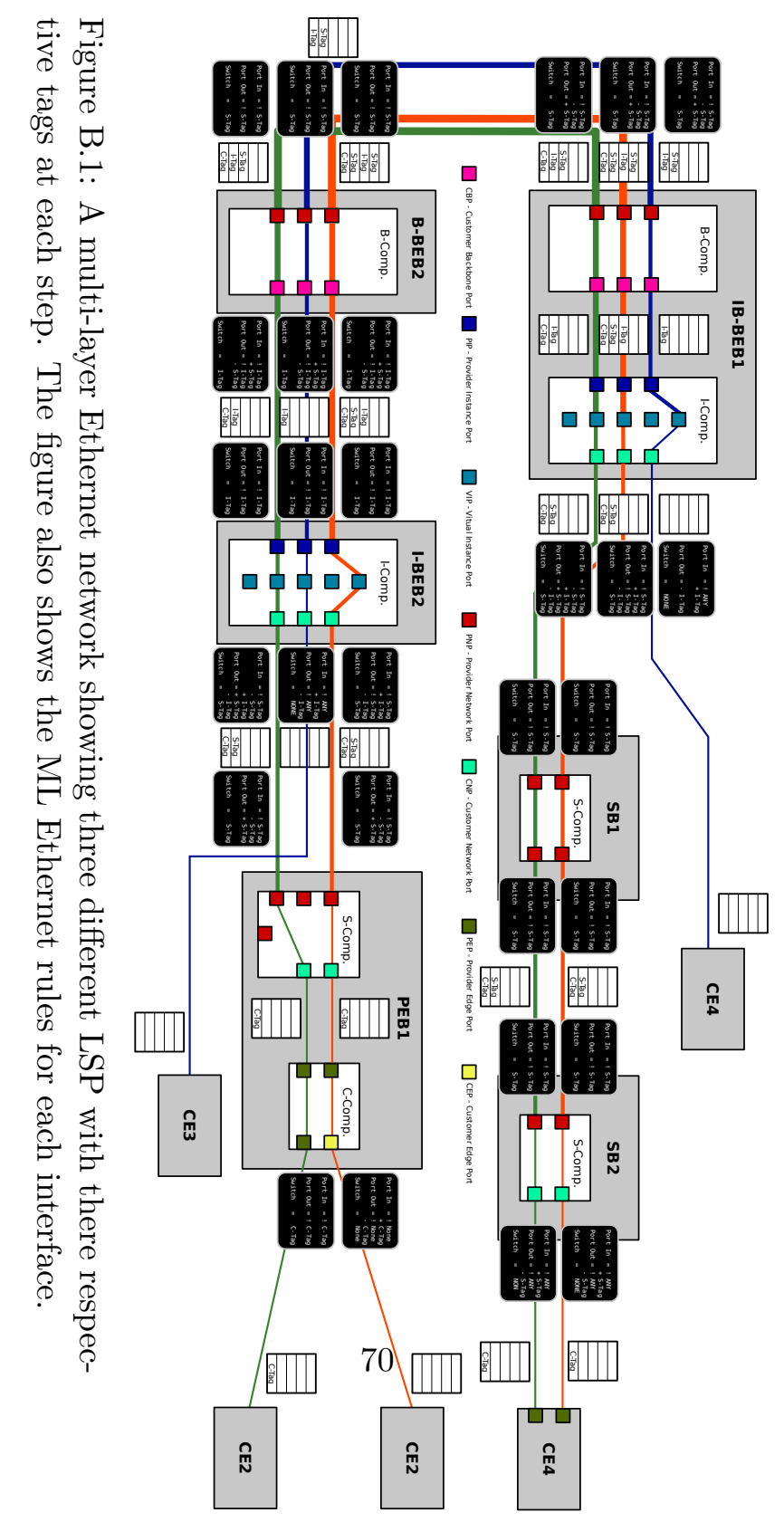

